\title{
Subepithelial Myofibroblasts Are Critical Regulators of Intestinal Epithelial Restoration
}

Vanesa Fernández-Majada ${ }^{1,7 \# *}$, Jordi Comelles ${ }^{1,6 \#}$, Verónica Acevedo ${ }^{1}$, Aina AbadLázaro ${ }^{1}$, Xavier Hernando-Momblona ${ }^{2,3}$, Eduard Batlle ${ }^{2,3,4}$, Elena Martinez ${ }^{1,5,6 *}$

${ }^{1}$ Biomimetic Systems for Cell Engineering Laboratory, Institute for Bioengineering of Catalonia (IBEC), The Barcelona Institute of Science and Technology (BIST), Baldiri Reixac 15-21, 08028 Barcelona, Spain

${ }^{2}$ Colorectal Cancer Laboratory, Institute for Research in Biomedicine (IRB Barcelona), The Barcelona Institute of Science and Technology (BIST), Baldiri Reixac 10-12, 08028 Barcelona, Spain

${ }^{3}$ Centro de Investigación Biomédica en Red (CIBERONC), Barcelona, Spain.

${ }^{4}$ ICREA, Passeig Lluís Companys 23, 08010 Barcelona, Spain

${ }^{5}$ Centro de Investigación Biomédica en Red (CIBER), Av. Monforte de Lemos 3-5, Pabellón 11, Planta 0, 28029 Madrid, Spain

${ }^{6}$ Department of Electronics and Biomedical Engineering, University of Barcelona (UB), Martí $i$ Franquès 1, 08028 Barcelona, Spain

${ }^{7}$ Department of Pathology and Experimental Therapeutics, University of Barcelona (UB), Feixa Llarga, 08907 l'Hospitalet de Llobregat, Spain.

\#These authors contributed to this work equally

*Sharing corresponding authorship. Contact information:

Biomimetic Systems for Cell Engineering Laboratory, Institute for Bioengineering of Catalonia (IBEC), Baldiri Reixac, 15-21, 08028 Barcelona, Spain

E-mail address: emartinez@ibecbarcelona.eu; vfernandez@ibecbarcelona.eu

Tel: +34934037177

Fax: +34 934034750

Short running title: Myofibroblasts assist epithelial cells to migrate. 


\begin{abstract}
Intestinal epithelial migration is supposed to occur in a passive manner driven by the mitotic pressure exerted either by the cryptal stem cells, under physiological conditions, or the newly formed epithelium, upon damage. However, whether interactions between different neighboring cell types and with the matrix contribute to epithelial movement remain elusive. Here, we developed a novel three-dimensional in vitro intestinal mucosa model of gap closure, that includes both the epithelium and the basement membrane cellular compartments in a spatially relevant manner, to show that intestinal subepithelial myofibroblasts (ISEMFs) play a crucial role in epithelial restoration. ISEMF-derived biochemical cues boost epithelial proliferation and maintain epithelial barrier integrity. While, at the wounded area, ISEMF actively migrate to the epithelial front where they activate and generate a-SMA contractile stress fibers along the direction of the epithelial cells promoting epithelial migration. Furthermore, ISEMF also deposit collagen paths that act as "guide rails" for directing IEC migration. Thus, the mere physical presence of ISEMFs can greatly accelerate restoration in wound healing, which suggests that ISEMFs should be recognized as new potential therapeutic targets.
\end{abstract}




\section{Introduction}

The small intestinal epithelium is formed by a monolayer of tightly packed and polarized epithelial cells arranged into crypt-villus units, in which one finger-like protrusion (villus) is surrounded by several epithelial invaginations (crypts) (Clevers, 2013; Leushacke and Barker, 2014). The intestinal epithelium is one of the fastest renewing tissues in mammals. This fast turn-over is fueled by the rapid division of the cryptal Lgr5 ${ }^{+}$intestinal stem cells (ISCs) which generate committed progeny that mature into different adult intestinal epithelial cell (IEC) types while following a rapid migratory path towards the villus tip where they undergo apoptosis and are spilled into the lumen (Barker et al., 2007; Barker, 2013). One of the main functions of the intestinal epithelium is to act as a physical protective barrier that prevents external insults from coming into contact with the immune system of the lamina propria. Any dysregulation of the normal epithelial homeostasis might cause the loss of intestinal epithelial barrier integrity. In such situations, an efficient restoration of the epithelium, including epithelial proliferation and migration, is critical to avoid the development of pathological inflammatory conditions that can lead to inflammatory bowel diseases (IBDs) or cancer development (Terzić et al., 2010; Karin and Clevers, 2016). Thus, intestinal epithelial migration is a crucial cellular process to maintain intestinal tissue homeostasis in both physiology and disease.

Not much is known about the contribution of mesenchymal cells in intestinal epithelial migration. In the adult intestine, IECs sit directly above the basement membrane, which is composed of a reticular collagen fibrils sheet and intestinal subepithelial fibroblasts, including myofibroblasts henceforth referred to as intestinal subepithelial myofibroblasts (ISEMFs) (Roulis and Flavell, 2016), and telocytes (Aoki et al., 2016). Based on morphology, ultrastructure, location and molecular markers, intestinal telocytes are in retrospect counterparts of myofibroblasts and therefore considered, by many authors, equivalent populations of subepithelial mesenchymal cells (McCarthy, Kraiczy and Shivdasani, 2020). ISEMFs form a syncytium that surround the crypts and villi of the intestine (Richman et al., 1987; Federici and Boulis, 1999; Powell et al., 2005; Mifflin et al., 2019). They are characterized by the expression of intracellular cytoskeletal a-smooth muscle actin (a-SMA), bundles of myofilaments and are interconnected via gap junctions that allow them to contract similarly to smooth muscle cells (Hinz et al., 2001; Eyden, 2008; Eyden, Curry and Wang, 2011; Powell et al., 2011; Roulis and Flavell, 2016). It is known 
that resident ISEMFs possess secretion capacities and, together with other subepithelial mesenchymal cells, act as ISC niche components by producing molecular gradients that regulate Wnt and BMP signaling along the crypt villus axis. (Powell et al., 2011; Roulis and Flavell, 2016; Shoshkes-Carmel et al., 2018; McCarthy et al., 2020). They have also been shown, in vitro, to support intestinal epithelial cell growth and differentiation through paracrine signals (Lahar et al., 2011; Lei et al., 2014). Resident myofibroblasts are also found in other epithelia such as in neonatal lungs (McGowan et al., 2008; Branchfield et al., 2016), where they contribute to the alveologenesis (Branchfield et al., 2016) and provide the lungs with elasticity and tensile strength (Toshima, Ohtani and Ohtani, 2004). However, the function of resident myofibroblasts in the physiological migration and the restoration of the homeostasis upon tissue damage in epithelial tissues is not very well understood.

Analyses carried out in epithelia such as the skin (Gabbiani, Ryan and Majno, 1971), cornea (Gorono et al., 1992; Jester, Petroll and Cavanagh, 1999) or cleaved palate (Chitturi et al., 2015), indicate that fibroblasts and myofibroblasts could contribute to epithelial restoration (Tomasek et al., 2002; Hinz, 2010). The wound healing process in these tissues is associated with the progressive activation of fibroblasts to myofibroblasts in response to mechanical stress and transforming growth factor $\beta 1$ (TGF- $\beta 1$ ) signalling (Desmouliere et al., 1993; Vaughan, Howard and Tomasek, 2000; Hinz et al., 2001). Activated myofibroblasts express a-SMA and develop stress fibers that exert contractile forces. These forces are directly transmitted to the ECM and induce the closure of the wound (Tomasek et al., 2002). In addition, activated myofibroblasts deposit collagen and express metalloproteases further contributing to the tissue matrix remodeling. However, it remains unclear whether these mechanisms occur in the intestine, and which is the specific role of the resident ISEMFs in promoting intestinal epithelial migration and restoration under both physiological and pathological conditions.

Here, we employ a three-dimensional (3D) intestinal mucosal model of gap closure to analyze the contribution of biochemical and mechanical ISEMF-derived signals to intestinal epithelial migration and restoration. Our 3D model includes primary IECs, primary ISEMFs, and the basement membrane matrix components, secreted by the ISEMFs, in a spatially relevant manner that mimics the tissue compartments in vivo. Our results indicate that ISEMFs are essential for a fast and efficient epithelial reconstitution. We show that ISEMs are important paracrine inducers 
of epithelial growth in our system. Furthermore, we prove that the physical presence of ISEMFs in the culture favors a directed epithelial migration. We demonstrate a coordinated migration with IECs and ISEMFs moving in a cooperative and guided manner, through their physical interaction, to facilitate the wound closure. Thus, we suggest resident ISEMFs as key regulators of active epithelial restoration of the intestinal epithelium through both paracrine and physical cues.

\section{Results}

Intestinal epithelial migration is impaired in the absence of a basement membrane cellular compartment

To study intestinal epithelial migration in a gap closure process using a physiologically relevant setup, we employed a method previously developed in our lab to grow intestinal epithelial cell monolayers (Altay et al., 2019). Briefly, we seeded intestinal organoid-derived single cells on hard tissue plates coated with a thin film of Matrige ${ }^{\circledR}($ Figure $1 \mathrm{~A})$. Cells adhered to the matrix and grew into a cobblestone-shaped epithelial monolayer that recapitulated the in vivo-like cell type and composition. They organized into proliferating crypt-like domains and differentiated villus-like regions (Altay et al., 2019) (Figure 1B). Furthermore, expression of the filamentous actin (F-actin) in the apical cell side and of the adherent junction protein $\beta$-catenin at the basolateral cell side demonstrate that the newly formed epithelial monolayers were mature and polarized (Figure 1B, low right panels). To perform gap closure studies, we attached elastomeric barriers to the substrate before seeding with cells to spatially control the growth of the epithelial monolayer. By removing the barrier this creates a cell-free gap in the middle of the tissue culture plate (Figure 1A). To track intestinal epithelial movement via live imaging we employed intestinal organoids, henceforth referred to as Lgr5-EGFP/RCL-tdT, which express high levels of tdTomato fluorescence in all cells (Figure 1 - Figure Supplement 1). With these tools we obtained live images to track intestinal epithelial movement for a period of $60 \mathrm{~h}$ after barrier removal (Video 1). For a successful gap closure, epithelial tissues need to migrate toward the gap maintaining tissue integrity until closure is achieved (Begnaud et al., 2016). In contrast, with our experimental setup the intestinal epithelial cells failed to close the gap. Specifically, the intestinal epithelial cells did not move in a cohesive manner and the migration front did not form properly. Instead, groups of 
cells appeared at the boundary between the tissue and the gap forming discrete protrusions (Figure $1 \mathrm{C}, \mathrm{t}=0 \mathrm{~h}$, white arrows). Initially, these protrusions were similar to the finger-like structures observed in other types of epithelia (Reffay et al., 2011). However, epithelial cells in between protrusions did not advance forward, and the finger-like structures became more elongated and started to lose their directional movement towards the gap (Figure 1C, $\mathrm{t}=30 \mathrm{~h}$ and $t=60 h$, white arrows). These abnormal advances of the leading edge led to the formation of holes in the sheet. Consequently, cells lost directionality and started to migrate back in the direction of these newly formed gaps (Figures 1C, D, white asterisks). Immunostaining analysis for $\beta$-catenin at different times after barrier removal confirmed the progressive loss of the epithelial tissue structure (Figure 1D). Thus, the intestinal epithelial gap closure is impaired when only epithelial cells are present in the culture.

A recent study proposed the existence of an actin-related protein $2 / 3$ complex-dependent active migratory force that would explain the physiological epithelial migration at the villi in vivo (Krndija et al., 2019). The appearance of protruding multicellular structures towards the gap in our results from an in vitro model of only intestinal epithelial cells would initially seem to support for this hypothesis (Figure 1). However, the failure in completing the closure of the gap indicates that the mere active migration together with the mitotic pressure arising from the crypt-like domains cannot account for the restoration of epithelial homeostasis. This suggests that other non-epithelial factors might be involved in this active epithelial restoration. Given the anatomical proximity of ISEMFs to IECs within the intestinal epithelium and their phenotypical and functional characteristics we decided to evaluate the potential of ISEMFs as epithelial migration regulators.

\section{Phenotypical and functional characterization of primary ISEMFs}

We adapted our epithelial crypt isolation protocol to further digest the basal lamina and obtain subepithelial fibroblasts. The obtained cells were then seeded onto tissue plates coated with a thin film of Matrigel ${ }^{\circledR}$ and their phenotype was analyzed using immunofluorescence. Cells stained positive for a-SMA and vimentin and negative for desmin, confirming their ISEMF identity and differentiating them from other subepithelial mesenchymal cells types (Figure 2A, left panels) (Lahar et al., 2011; Lei et al., 2014; McCarthy, Kraiczy and Shivdasani, 2020). Furthermore, primary isolated ISEMFs produced and expressed fibronectin, laminin, and collagen IV, thus 
confirming their ECM-protein secretory capacities (Figure 2A, right panels). This protein expression profile was maintained when cells were seeded onto non-coated plastic tissue plates, demonstrating the cell-specific expression of the cited proteins (Figure 2 - Figure Supplement 1 A and B). One of the most relevant characteristics of myofibroblasts is the development of a-SMA stress fibers that connect with the ECM at focal adhesion sites and between cells via adherent junctions (Hinz et al., 2007). Higher magnification images clearly demonstrated the expression of a-SMA in stress fibers (Figure 2B). These have been correlated with the production of isometric tension (Harris, Stopak and Wild, 1981), and provide myofibroblasts with stronger contractile capacities compared to regular fibroblasts (Vaughan, Howard and Tomasek, 2000; Hinz et al., 2001; Hinz and Gabbiani, 2003). To analyze the ability of our ISEMFs to contract, we seeded them on floating collagen discs and checked for their contraction, comparing ISEMFs to non aSMA-expressing fibroblasts (NIH-3T3) and epithelial cells (Caco-2), which served as negative controls. Primary ISEMFs reduced the area of the collagen discs by about $80 \%$ after one day in culture while NIH-3T3 and Caco-2 only achieved a reduction of $20 \%$. While this difference was slightly reduced after 2 days in culture, it still showed a more than twofold stronger contractile capacity of the ISEMFs compared to regular fibroblasts (Figure 2C), as previously shown (Hinz et al., 2001). Overall, this characterization confirms the ISMEFs-typical cell morphology, markers expression, protein secretion and contractility capacities of our primary isolated intestinal subepithelial cells.

\section{ISEMFs boost epithelial proliferation through paracrine signals}

We then evaluated the impact of ISEMFs on intestinal epithelial growth by performing 3D Matrige $\left.\right|^{\circledR}$ drop co-cultures of crypts and ISEMFs and observing the evolution of the crypt's growth and morphology for 4 consecutive days. To disentangle the ISEMF-derived paracrine signals from the crypt-ISEMF physical interactions on intestinal epithelial cell growth, we compared crypts cultured with basic medium, cultured with ISEMF-derived condition medium (ISEMF_CM), and crypts co-cultured with ISEMFs in direct physical contact. For comparison we also added cultures in which the ISEMFs were present but physically separated from the Matrige ${ }^{\circledR}$ drop containing the crypts by using a Transwell ${ }^{\circledR}$ insert (Figure 3A, schematics). Our results show that when crypts are cultured with medium conditioned by the ISEMFs (either with ISEMF_CM or with the ISEMFs seeded in Transwell ${ }^{\circledR}$ inserts), they form epithelial cysts. This contrasts with the normal budding 
and differentiated organoids formed when crypts are cultured with the basic medium (Figure 3A, first three columns). Those intestinal cysts are hollow epithelial spheres mainly composed of Ki67 proliferative cells (Figure 3B, C). They closely resemble crypts with hyperactivation of the Wnt pathway (Sato et al., 2011; Drost et al., 2015; Jardé et al., 2018). Indeed, it has been shown that ISEMFs produce Wnt molecules (Gregorieff et al., 2005; Farin, Van Es and Clevers, 2012; Kabiri et al., 2014). Thus, ISEMFs might boost epithelial proliferation and cyst formation through the paracrine activation of the $\beta$-catenin signaling pathway. Interestingly, intestinal crypts grown in direct contact with ISEFMs also form cysts which grow through a series of contraction-relaxation pulsatile cycles (Video 2). Those cysts are bigger and not as spherical as those formed with only ISEMFs paracrine signals. (Figure 3A, last column). In addition, we observed that ISEMFs physically interact with the intestinal cysts, pulling them closer together until eventually causing their contact and fusion to create bigger structures (Figure 3D and Video 3). Over time, those fused cysts evolve into flattened structures corresponding to rudimentary epithelial layers (Figure3D, right panel). a-SMA immunostaining revealed the presence of ISEMFs at the protruding edges of those epithelial monolayers (Figure 3E). ISEMFs appear to be pulling on the epithelial cells causing them to stretch and move (Figure 3E). Overall, these results suggest that primary ISEMFs boost intestinal epithelial proliferation through paracrine signals and reveal a key contribution of ISEMFs in regulating epithelial movement through epithelial-myofibroblast direct physical interactions.

\section{The physical presence of ISEMFs is crucial for an efficient and directed epithelial migration}

To further analyze the paracrine and physical effects of ISEMFs on intestinal epithelial movement, we employed our 3D intestinal mucosa model of gap closure to compare the capacity of intestinal epithelial cells to migrate and close a cell-free gap in the presence of (i) only ISEMF-derived paracrine signals and (ii) when ISEMFs are physically present in a spatially relevant manner in the culture (Figure 4A, schematics). To analyze scenario (i) we seeded intestinal epithelial cells onto a thin layer of Matrigel $^{\circledR}$ and cultured them with ISEMF_CM. For the scenario (ii), we first grew a layer of ISEMFs on a thin layer of Matrigel ${ }^{\circledR}$ (emulating the basement membrane cellular and matrix compartments) and then seeded the intestinal epithelial cells on top to form the epithelial monolayer. IECs grown onto a thin layer of Matrigel cultured with basic medium were used as control. We again employed intestinal epithelial cells derived from Lgr5-EGFP/RCL-tdT 
intestinal organoids to track cells by time-lapse microscopy. Right after the removal of the barrier, IECs formed comparable epithelial monolayers for all the conditions tested (Figure $4 \mathrm{~A}, \mathrm{t}=0 \mathrm{~h}$ ). We tracked the trajectories of individual IECs for each condition for almost 2 days (45h). Clearly, IECs exhibit a more uniform and directed movement in the direction of the cell-free gap when grown on top of a layer of ISEMFs (Figure 4A, right panels; Video 4) than in the absence of ISEMFs (Figures $1 \mathrm{C}$ and 4A, left panels; Video 5). Notice that, for longer recording times $(t=60 \mathrm{~h})$, this resulted in a complete gap closure when IECs were grown on top of a continuous layer of ISEMFs (Figure 4 - Figure Supplement 1 A). Interestingly, complete gap closure did not happen when IECs were exposed to ISEMFs paracrine signals only. In this case, although IECs migration was improved, their movements were still disorganized and the monolayer lost cohesiveness forming new gaps, although smaller than the sample with basic medium (Figure 4A, middle panels; Video 4). Results from the $\beta$-catenin immunostainings seem to support these findings as only epithelial monolayers grown on top of ISEMFs remained intact after 45h of cell migration. Meanwhile, ISEMF_CM was insufficient to maintain a packed and cohesive epithelial monolayer during the migration process (Figure 4 - Figure Supplement $1 \mathrm{~B}$ ). These results demonstrate that the physical interaction between ISEMFs and IECs is important for promoting epithelial migration while maintaining optimal tissue organization.

To further characterize the effect of ISEMFs presence on IECs trajectories, we tracked individual cell trajectories during $45 \mathrm{~h}$ after removing the elastomeric barrier. In the control sample, the trajectories of IECs were poorly oriented towards the gap (Figure 4B, left panel), because of the lack of integrity of the epithelia during gap closure and the appearance of newly formed gaps. Although the paracrine effect of ISEMFs improved the IECs directionality, only the physical presence of the ISEMFs underneath the IECs completely oriented the cell trajectories toward the gap (Figure 4B, middle and right panels). We also measured (i) the net displacement and (ii) the direction with respect to the gap for each of the trajectories (Figure 4C). The net displacement is defined as the vector between the initial and final point of each trajectory, regardless of the trajectory in-between. Compared to the control condition, the average net displacement was increased as a response to the ISEMFs signaling, either paracrine or physical (Figure 4 - Figure Supplement 2 B). Noteworthy, despite being the overall amount quantitatively undistinguishable, the net displacement pattern of IECs as a function of their position within the epithelia was 
different for cells with only ISEMF-derived paracrine signals than for those with also the physical presence of ISEMFs (Figure 4D). ISEMF-derived paracrine signaling induced a progressive increase of the cell net displacements for cells closer to the migration front (gray stripes in the graphs of Figure 4D). However, this increase was enhanced when ISEMFs were physically present, retrieving the typical velocity profiles of in vitro epithelial expansion (Lee et al., 2017). Furthermore, to determine if the net displacement of cell trajectories was performed towards the gap, we plotted the distribution of the angles between the net displacement vector and the gap direction (Figure 4C and Figure 4 - Figure Supplement $2 \mathrm{C}$ ). The angular distribution of these directions (Figure 4 - Figure Supplement 2 C) confirmed that the directional bias towards the wound (peaked around $0^{\circ}$ ) was dependent on whether epithelial cells were exposed to paracrine signals of ISEMFs or to their physical presence. While 35\% of trajectories on the control sample displayed $-20^{\circ}<\alpha<20^{\circ}$, this increases up to $47 \%$ for samples cultured with ISEMF-derived paracrine signals and up to $70 \%$ (twofold with respect to the control) by the physical presence of the ISEMFs underneath the epithelia. As for the net displacement, we analyzed the alignment of individual cell trajectories with the gap direction as a function of the cell distance to the migrating front. To ease the comparison, we defined and alignment index $(\cos (2 \cdot \alpha))$ that equals 1 when the net displacement is in the direction of the gap and -1 when it is perpendicular. We found that the physical presence of ISEMFs increases dramatically such alignment within the first $250 \mu \mathrm{m}$ away from the migrating front (Figure 4E). Moreover, when comparing the trajectories among the three conditions within the first $250 \mu \mathrm{m}$ (red rectangle in Figure 4 - Figure Supplement 2 D), gaporiented tracks progressively increase in paracrine and physical conditions. But, only when ISEMFs are physically present the vast majority of trajectories are aligned (Figure 4E and Figure 4 - Figure Supplement 2 E). On the contrary, deeper into the epithelia, the mean value of the alignment index decreases, and the standard deviation increases (shadowed area of Figure 4E) in a similar manner for the three conditions, suggesting a broader distribution of trajectory directions. Overall, these results show a spatially dependent enhancement of the directionality and displacement of the epithelial cells close to the migration front when ISEMFs are physically present in the culture. This points toward the existence of physical interactions between the IEC and ISEMFs that regulate epithelial migration and make it more efficient. 
ISEMFs accumulate and align with IECs at the epithelial migration front for an active and collaborative epithelial migration toward the gap.

To better characterize the physical IEC-ISEMF interaction during epithelial migration, we analyzed by immunofluorescence the progression of the co-culture from the moment of the barrier removal until the closure of the gap. We utilized a-SMA antibodies to specifically visualize ISEMFs' morphology and actin stress fibers, and $\beta$-catenin antibodies to visualize the structure of the epithelial monolayer. We found that if ISEMFs were seeded on a thin layer of Matrigel ${ }^{\circledR}$ formed a continuous mesenchymal layer of a-SMA ${ }^{+}$cells (Figure 5A, Day 2). Although neighboring ISEMFs could align locally, cells did not exhibit any preferred directionality and they were fairly evenly distributed throughout the plate. Once the epithelial monolayer had formed on top of the ISEMFs layer, immediately after removing the elastomeric barrier, the ISEMFs distribution was not altered (Figure 5A, Day 4). However, alignment and directionality toward the gap was starting to appear among cells, especially near the epithelial front (Figure 5A, Day 4). In order to quantify this observation, we measured the dominant directions of the ISEMF monolayers and of the IEC epithelia. We subdivided the images in subregions of roughly the size of an intestinal epithelial cell. Then, we extracted the dominant direction $(\omega)$ within each subregion based on the spatial gradient of the fluorescent signal (see the methods section for detailed explanation). We used the signal of $\beta$-catenin for IECs and of $\alpha$-SMA for ISEMFs, obtaining the dominant direction for each cell type (Figure 5B). We then measured the angle $\theta$ between the ISEMFs' dominant direction and the IECs' dominant direction in each subregion. By plotting the value of $\cos (2 \cdot \theta)$ (which we referred as correlation index (Figure 5B)) for each subregion we could generate correlation maps of alignment between the two cell types (note that regions where cells did not have a well-defined dominant direction did not contribute to the correlation maps (see the methods section for details)). Correlation analysis showed that only regions adjacent to the migration front had dominant (albeit only weakly correlated) direction right after removing the stencil (Figure 5B, Day 4 and Figure 5 - Figure Supplement 1). This correlation increased with time and by Days 5 and 6 the epithelial migration front had moved towards the gap and the number of ISEMFs near the gap had also increased. Also, the directionality had improved at this point with the ISEMFs appearing more elongated and with clearly visible a-SMA ${ }^{+}$stress fibers (Figure 5A, Days 5 and 6). Furthermore, the correlation between the orientations of ISEMFs and 
IECs increased (the correlation map became predominantly yellow), as both cell types became increasingly parallelly aligned (Figure 5B, Days 5 and 6). Such alignment between both cell types progressively moved from the epithelial front to deeper into the epithelium along time (Figure 5B, Days 5 and 6). Thus, during the early stages of the migration process, i.e., immediately after removing the elastomeric barrier (Day 4), the region close to the migration front shows small regions of elevated correlation/alignment between ISEMFs and IECs (Figure 5 - Figure Supplement 1). As time progresses, the size of these regions and the correlation/degree of alignment increases. While on Day 5 the correlation maximum was still confined to the migration front, just one day later it already extended further into the epithelium (Figure 5 - Figure Supplement 1). Noteworthy, the accumulation of ISEMFs, with high levels of a-SMA stress fibers, and the alignment between the ISEMFs and IECs at the epithelial migration front coincided temporally and spatially with the increased directionality of the epithelial trajectories towards closing the gap (Figure 4E and Figure 4 - Figure Supplement $2 \mathrm{D}$ and $\mathrm{E}$ ). This fact clearly suggests an active and collaborative process between the two cell types to achieve a more efficient epithelial migration.

ISEMFs and IECs orient with the direction of the gap and migrate following ISEMF-secreted collagen paths

In order to shed some light on how this active and collaborative migration might occur, we first analyzed the onset of gap closure (first 9 hours after removing the stencil) by particle image velocimetry (PIV). This allowed us to obtain velocity fields from the phase contrast movies of the migration fronts, which comprise both the IECs and the ISEMFs covering the gap. Strikingly, two clearly differentiated regions appeared on the maps of the x-component of the mean velocities $\left(v_{x}\right)$ (Figure $\left.6 A\right)$. The region at the left of the migration front (corresponding to IECs) exhibited on average $v_{x}>0$, which corresponds to cells migrating toward the gap. In contrast, the region at the right of the migration front (corresponding to ISEMFs) exhibited on average $v_{x}<0$, which correspond to cells migrating from the gap toward the epithelial front. When looking at the temporal evolution of the $\mathrm{x}$-component of the instantaneous velocity (Figure 6B), we observed the expansion of the $v_{x}>0$ region toward the gap, which matches with the advancing of the epithelia. Furthermore, the averaged profiles of the x-component $\left(v_{x}\right)$ and the $y$-component $\left(v_{y}\right)$ of the velocities across the migration fronts and the adjacent gap regions (Figure 6C) showed that 
migration occurred mainly in the direction perpendicular to the front for both cell types. In the case of the epithelial region, $v_{x}$ was positive and $v_{y}$ was zero in agreement with the results obtained by manually tracking individual cells (Figure 4B). In the case of the region associated to the ISEMFs (the gap region), $v_{x}$ was negative and $v_{y}$ was zero, which suggests that the myofibroblasts actively migrated toward the front of the migrating epithelia. Then, we analyzed the average cell orientation of both ISEMFs and IECs relative to the direction of the gap. On Day 4, the ISEMFs tended to be oriented perpendicular to the gap. Just one day later, most ISEMFs exhibited an orientation parallel to the gap (Figure 6D, lower graph). This became more random by Day 6 , especially far from the migration front. In contrast, the IECs maintain their mean orientation slightly parallel with the gap throughout (Figure 6D, upper graph), while the number of cells oriented perpendicular with the gap is reduced along time. Altogether, these suggest that the ISEMFs may be an active cell component of the culture in the gap closure process. On the one hand, by actively migrating toward the front of the epithelia and, on the other hand, by modifying their orientation to be aligned with the IECs and parallel with the gap to make the epithelial migration more efficient.

We then reasoned that ISEMFs might contribute to the epithelial migration by secreting ECMproteins. We found that the secretion of collagen IV and its accumulation increased with time (Figure 6E). Interestingly, we observed ordered collagen-path depositions accumulating between the ISEMFs layer and the epithelium (Figure 6F, 6G), in a similar fashion to the observed ISEMFs preferential orientations. Taken together, these results suggest that when the epithelial migration starts, ISEMFs expressing a-SMA ${ }^{+}$stress fibers actively align with the IECs and orient with the gap at the epithelial migration front. These collagen-paths deposited by ISEMFs below the IECs might serve as tracks for the epithelial movement. In combination with the increased directionality of epithelial migration close to the front, our data indicate that epithelial movement toward the gap closure is more effective and directionally guided by the physical presence of the underneath ISEMFs.

\section{Discussion}

We employed a 3D in vitro intestinal model of gap closure to analyze the effect of the resident population of ISEMFs on epithelial restoration. We could show that ISEMF-derived paracrine signals regulate epithelial proliferation. According to literature, this might happen through the secretion of non-epithelial Wnt pathway activators including Wnt2, Wnt2b, Wnt4, Wnt5a, Wnt9b 
and Wnt11 (Gregorieff et al., 2005; Farin, Van Es and Clevers, 2012; Kabiri et al., 2014), and the enhancers of the Wnt pathway, R-spondin 2 and R-spondin 3 (Kim et al., 2008; Kabiri et al., 2014; Lei et al., 2014). All these have shown to be factors secreted by fibroblasts that potentiate intestinal stem cell activity by paracrine action, and our results indicate that they also improved epithelial migration. However, we show that only ISEMFs physical presence allows for the complete epithelial restoration in a process that involves ISEMFs active migration, expression of a-SMA ${ }^{+}$stress fibers and reorientation with the gap as soon as the migration process starts. This suggest that resident ISEMFs might act as a platform (similar to a conveyor belt), which through their contractile properties, and capacity to generate forces they could mechanically stimulate epithelial cells to move in a certain direction. In addition, we could show that ISEMFs deposit collagen paths which could act as "guide rails" for migrating intestinal epithelial cells. This phenomenon seems reminiscent of how cancer associated fibroblasts (CAFs) promote tumor invasion by creating ECM "highways" that tumor cells use to escape from the tumor bulk (Gaggioli et al., 2007; Attieh et al., 2017; Gopal et al., 2017). Whether CAF-derived mechanical signals also contribute to tumor cell mobility is still unclear, although it seems possible considering that CAFs, similarly to ISEMFs, are also characterized by an activated, highly contractile phenotype (Turley, Cremasco and Astarita, 2015).

Intestinal epithelial migration, both under physiological and pathological conditions is thought to happen passively pushed by the stem cell division at the bottom of the crypts or by the proliferation of the newly formed epithelium upon damage (Parker et al., 2017). Our results present a counterpoint to this idea by introducing the concept of ISEMF-derived physical signals facilitating epithelial active migration. Despite not having been proved in vitro before, this is not the first time that passive intestinal epithelial migration, is being challenged. In a recent publication, Krndija et al. elegantly demonstrated that physiological epithelial migration along the intestinal crypt-villus axis cannot be explained by only the mitotic pressure exerted by stem cells at the bottom of the crypts. To do that, they combined in vivo genetic mouse models with biophysical modeling. They proposed that, while mitotic pressure action is constrained mainly to the crypts, epithelial migration in the villus could be an active migration process, determined by an actin-related protein 2/3 complex (Krndija et al., 2019). Given our results, it would be interesting to analyze the contribution of ISEMF-derived physical cues for regulating the active physiological epithelial 
migration along the intestinal crypt-villus axis. A pioneering work in the field that dates back more than 50 years analyzed the colonic ISEMF dynamics in vivo via ${ }^{3} \mathrm{H}$-timidine auto-radiographic examination. Authors proved that ISEMFs actually migrate along the walls of the crypts, and reach their tops in an equivalent time that the employed by the epithelial cells, thus indicating an intimate relationship between both cell types and a possible synchronous migration (Pascal, Kaye and Lane, M.D, 1968), analogous to the conveyor belt principle. This work also revealed localized arrangements of collagen fibrils underneath the epithelium in a fairly organized arrangement, compared to the looser collagen network present within the rest of the lamina propria (Pascal, Kaye and Lane, M.D, 1968). This would favor the notion, presented here, that collagen paths built by the ISEMFs guide for epithelial movements. In this context, a 3D intestinal scaffold harboring primary IECs, a basement membrane and ISEMFs grown in a spatial physiologically relevant manner would represent the perfect in vitro tool to study the effects of cell-to-cell and cell-to-matrix interactions on regulating physiological intestinal epithelial migration.

In addition to be a major organ involved in digestion, the intestinal epithelium is a protective physical barrier that prevents external insults to come into contact with the lamina propria immune system. Specific genetic conditions, external pathogens, and physical or chemical agents might induce a loss of integrity of this epithelial barrier. Once it occurs, a fast and efficient repair of the epithelial damage is crucial for maintaining intestinal homeostasis and preventing uncontrolled inflammatory responses (Terzić et al., 2010; Karin and Clevers, 2016). Thus, understanding the mechanisms that regulate intestinal epithelial repair upon intestinal damage is critical to design new avenues of inflammatory bowel diseases and cancer therapies. Here, we proposed a new functionality for ISEMFs, acting as important players in intestinal epithelial restoration by regulating both epithelial proliferation and migration, which should prompt their recognition as new potential therapeutic targets.

\section{Material and Methods}

\section{Mouse models}

All experimental protocols involving mice were approved by the Animal care and Use Committee of Barcelona Science Park (CEEA-PCB) and the Catalan government and performed in accordance with their relevant guidelines and regulations. Lgr5-EGFP-IRES-creERT2 mice have 
been previously described (Barker et al., 2007). Briefly, Lgr5-EGFP-IRES-creERT2 mice were generated by homologous recombination in embryonic stem cells targeting the EGFP-IREScreERT2 cassette to the ATG codon of the stem cell marker Lgr5 locus, allowing the visualization of $\mathrm{Lgr}^{+}$stem cells with a green fluorescent protein (GFP). Lgr5-EGFP-IRES-creERT2 mice were crossed with the Cre reporter strain Ai9 (RCL-tdT) (JAX-007909) to generate the Lgr5-EGFPIRES-creERT2/RCL-tdT mouse. Ai9 (RCL-tdT) is designed to have a loxP-flanked STOP cassette preventing transcription of the CAG promoter-driven red fluorescent variant (tdTomato), inserted into the Gt(ROSA)26Sor locus. Upon Cre-mediated recombination Lgr5-EGFP-IREScreERT2/RCL-tdT mice express robust tdTomato fluorescence in Lgr5-expressing cells and their progeny.

\section{Intestinal crypts isolation and culture}

Intestinal crypts from Lgr5-EGFP-IRES-creERT2/RCL-tdT mice were isolated as previously described (Fernández-Majada et al., 2016; Barriga et al., 2017). Briefly, small intestines were flushed with PBS and cut longitudinally. Villi were mechanically removed, and intestinal crypts were isolated by incubating the tissue with PBS containing 2 mM EDTA (Sigma) for 30 minutes at $4^{\circ} \mathrm{C}$. The digestion content was filtered through a $70 \mu \mathrm{m}$ pore cell strainer (Biologix Research Co.) to obtain the crypt fraction. Crypts were plated in Matrige ${ }^{\circledR}$ (BD Bioscience) drops and supplemented with basic medium: advanced DMEM/F12 (Invitrogen) plus 1\% Glutamax (Gibco), 1\% HEPES (Sigma), Normocin (1:500, Invitrogen), 2\% B27 (Gibco), 1\% N2 (Gibco), 1.25 mM Nacetylcysteine (Sigma), supplemented with recombinant murine EGF (100 $\mathrm{ng} \mathrm{ml}^{-1}$, Gibco), recombinant human R-spondin 1 (200 $\mathrm{ng} \mathrm{ml}^{-1}$, R\&D Biosystems), and recombinant murine Noggin (100 $\mathrm{ng} \mathrm{ml}^{-1}$, Peprotech), CHIR99021 (3 $\mu \mathrm{M}$, Tebu-bio) and valproic acid (1 mM, sigma) to formulate the ENR_CV-medium (Yin et al., 2014). The medium was changed every 2 to 3 days. The first 4 days of culture the Rho kinase inhibitor Y-27632 (Sigma) was added to the culture. Outgrowing crypts were passaged once a week and organoid stocks were maintained for up to 4 months.

\section{Generating Lgr5-EGFP/RCL-tdT intestinal organoids by in vitro tamoxifen dependent creERT2 induction}


Lgr5-EGFP-IRES-creERT2/RCL-tdT small intestinal organoids were treated in vitro with $100 \mathrm{nM}$ of 4-Hydroxitamoxifen (4-HT) (Sigma) for $48 \mathrm{~h}$ to induce the expression of tdTomato in $\mathrm{Lgr}^{+}$cells. Treated intestinal organoids were mechanically and enzymatically digested as described above and cell sorted to obtain pure $\mathrm{GFP}^{+}$and tdTomato ${ }^{+}$cell populations. Sorted cells were cultured in

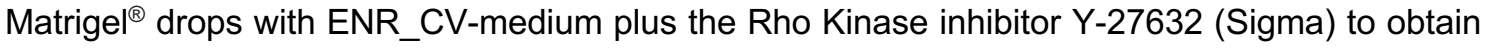
a new in vitro line of intestinal organoids named Lgr5-EGFP/RCL-tdT, which expresses a robust tdTomato fluorescence in all cells and GFP fluorescence in $\mathrm{Lgr}^{+}$stem cells. Outgrowing crypts were passaged once a week and organoid stocks were maintained for up to 4 months.

Intestinal organoid digestion to single cells

To obtain organoid-derived intestinal epithelial cells (IECs), organoids were first obtained from Lgr5-EGFP-IRES-creERT2/RCL-tdT mice. Then, fully-grown organoids were subjected to a digestion protocol. Briefly, Matrige ${ }^{\circledR}$ drops containing organoids were disrupted by pipetting with TrypLE Express1X (Gibco) and transferred to a Falcon tube at $4^{\circ} \mathrm{C}$, where mechanical disruption was applied using a syringe with a 23 G 1" needle (BD Microlance 3), was applied. Next, disrupted organoids were further digested by incubating them for 5 to 7 minutes at $37^{\circ} \mathrm{C}$ with vigorous handshaking every minute. Successful digestion to single cells was confirmed via inspection under the microscope.

\section{Intestinal subepithelial myofibroblast isolation and culture}

Intestinal subepithelial myofibroblasts (ISEMFs) were isolated from mouse intestine using a modified version of a previously reported protocol (Khalil et al., 2013). Briefly, left over tissue from the crypt isolation procedure (see above) was further digested by incubating at $37^{\circ} \mathrm{C}$ for $60 \mathrm{~min}$ at $200 \mathrm{rpm}$ with $2000 \mathrm{U}$ of collagenase (Sigma). The digested tissue was then pelleted and lysed at $47^{\circ} \mathrm{C}$ for 5 minutes using ACK lysis buffer (Gibco). The pellet, containing lamina propria cells, was resuspended in Dulbecco's modified medium (DMEM) (Life Technologies) containing 10\% foetal bovine serum (FBS) (Gibco), 1\% penicillin/streptomycin (Sigma), and 1\% minimum essential medium non-essential amino acids (MEM-NEAA) (Gibco) and cultured in tissue culture plates $\left(25 \mathrm{~mm}^{2}\right)$. After 1 week in culture, only lamina propria fibroblasts, mainly myofibroblasts, 
remained attached. Plates reached confluence after approximately 20 days in culture. Cell division of primary myofibroblasts is limited and after $6-8$ passages the cells become senescent. Detection by immunofluorescence of $\alpha$-smooth muscle actin ( $\alpha$-SMA), vimentin, and reduced desmin expression was used to assess the purity of the myofibroblast cultures.

\section{Intestinal subepithelial myofibroblast conditioned medium (ISEMF_CM) preparation}

For harvesting the intestinal subepithelial myofibroblast conditioned medium (ISEMF_CM), plates at 60 to $80 \%$ of confluence were maintained in culture for 6 days. Medium was harvested, centrifuged, and filtered using a $22 \mu \mathrm{m}$ pore filter (Millipore) and frozen until use. Medium harvested on different days but from the same myofibroblast isolation was pooled to have the same medium in all experiments. ISEMF_CM was complemented with $1 \%$ Glutamax, $1 \%$ HEPES, Normocin (1:500), 2\% B27, 1\% N2, $1.25 \mathrm{mM} \mathrm{N}$-acetylcysteine, EGF (100 $\left.\mathrm{ng} \mathrm{ml}^{-1}\right)$, human Rspondin $1\left(200 \mathrm{ng} \mathrm{ml}^{-1}\right)$, Noggin $\left(100 \mathrm{ng} \mathrm{ml}^{-1}\right)$, CHIR99021 (3 $\left.\mu \mathrm{M}\right)$, and valproic acid (1 mM) before use for organoid cultures.

\section{Setup of the 3D intestinal mucosa model to study epithelial migration in vitro (gap closure model)}

Ibidi $\mu$-Slides 8 well (Ibidi $\mathrm{GmbH})$ were coated with Matrige ${ }^{\circledR}$ to form thin $(<20 \mu \mathrm{m})$ films by spreading $10 \mu \mathrm{L}$ of Matrige ${ }^{\circledR}$ at $3 \mathrm{mg} \mathrm{ml}^{-1}$ throughout each well, followed by incubation at $37^{\circ} \mathrm{C}$ for 1 hour as previously described (Altay et al., 2019, 2020). 2\% Pluronic ${ }^{\circledR}$ F-68 (Sigma) treated elastomeric barriers (Polydimethylsiloxane (PDMS) stencils) of approximately 2,5 x $10 \mathrm{~mm}$ were used to spatially confine cell growth and generate an epithelial migration gap in the middle of the well. Different experimental conditions were analyzed: control (only IECs were present in the culture), paracrine (IECs were culture with ISEMF_CM (see SI Methods)) and physical (IECs and ISEMFs were co-cultured in physical contact). For the control samples, first elastomeric barriers were placed (using PDMS grease, Corning) on the Matrige ${ }^{\circledR}$ coated wells, horizontally dividing the well into two parts. Then, $3 \times 10^{5} \mathrm{IECs} \mathrm{cm}^{-2}$ were seeded and cultured with ENR_CVmedium containing Y-27632 for 2 days. For the paracrine samples, we employed the same seeding protocol than before, but using ISEMF_CM/ ENR_CV-medium containing Y-27632 for 2 days. For the physical samples, first $10^{5}$ ISEMFs $\mathrm{cm}^{-2}$ were seeded on Matrige ${ }^{\circledR}$ coated wells and were cultured with complete DMEM medium for 1 day. Then, the elastomeric barriers were placed 
onto the ISEMF monolayers and $3 \cdot x 10^{5} \mathrm{IECs} \mathrm{cm}^{-2}$ were seeded on top and cultured with ENR_CV-medium containing Y-27632 for 2 days more. For physical samples (discontinuous ISEMFs layer), first PDMS Stencils were placed on Matrige $\mathrm{I}^{\circledR}$ coated wells, next $10^{5}$ ISEMFs cm-

${ }^{2}$ were seeded and cultured with complete DMEM medium for 1 day; then $3 \cdot \times 10^{5} \mathrm{IECs} \mathrm{cm}^{-2}$ were seeded on top and cultured with ENR_CV-medium for 2 additional days. After the indicated tissue culture times, the elastomeric barriers were carefully removed using self-closing tweezers to avoid the detachment of the underlying cellular or Matrige ${ }^{\circledR}$ layer. Epithelial growth and migration to reepithelize the gap were monitored using time-lapse microscopy, conducted at 10x magnification with an Axio Observer 7 epifluorescence inverted microscope (Zeiss) employing temperature $\left(37^{\circ} \mathrm{C}\right)$, relative humidity $(95 \%)$, and $\mathrm{CO}_{2}(5 \%)$ regulation. Phase contrast and Alexa 546 channels were used. Images were acquired every $10 \mathrm{~min}$ up to $48 \mathrm{~h}$ of culture. The time-lapse videos and fluorescence images were processed with Fiji (http://rsb.info.nih.gov/ij, NIH, USA). For an optimal tracking of individual Lgr5-EGFP/RCL-tdT IECs (tdTomato') those were mixed and seeded with non-fluorescent IECs in a 1:0.5 ratio.

\section{Immunostaining}

Cells were fixed with $10 \%$ neutralized formalin (Sigma), permeabilized with $0.5 \%$ Triton X-100 (Sigma) and blocked with a blocking buffer containing 1\% BSA (Sigma), 3\% donkey serum (Millipore), and $0.2 \%$ Triton X-100 in PBS for at least 2 hours. Primary antibodies used were: anti$\beta$-catenin (1:100, Abcam), anti- collagen IV (1:250, BioRad), anti-Ki67 (1:100, BD Biosciences), anti- a-SMA (1:100, Sigma), anti- desmin (1:100, Abcam) and anti-vimentin (1:100, Developmental Studies Hybridoma Bank). All samples were incubated overnight with the primary antibodies at $4^{\circ} \mathrm{C}$ followed by $2 \mathrm{~h}$ incubation at room temperature (RT) with secondary antibodies Alexa Fluor 568 and Alexa Fluor 488 donkey anti-goat, and Alexa Fluor 647 (Jackson ImmunoResearch) diluted at 1:500. Nuclei were stained with 4',6-diamidino-2-phenylindole (DAPI, Life Technologies) (1:1000). Alexa Fluor 568 phalloidin (Tebu-Bio) was used to stain filamentous actin (F-actin). The fluorescence images were acquired using either a confocal laser scanning microscope (LSM 800, Zeiss) or an epifluorescence microscope (Axio Observer 7, Zeiss). The bright field images were obtained with an inverted fluorescence microscope (Eclipse Ts2, Nikon). 


\section{Collagen contraction gel assay}

Collagen type I from rat tail (Corning) was neutralized with $\mathrm{NaOH}$, made isotonic with 10X PBS and diluted to a final concentration of $3 \mathrm{mg} \mathrm{ml}^{-1}$ on ice. For these experiments we employed: primary intestinal subepithelial myofibroblasts (ISEMFs), NIH-3T3 fibroblasts (ATCC 8 CRL$1658^{\mathrm{TM}}$ ) and Caco-2 cells (ATCC $®$ HTB- $37^{\mathrm{TM}}$ ). Cells were trypsinized following standard methods, counted, and mixed with the neutralized collagen I solution on ice. Approximately 200 $\mu \mathrm{l}$ of collagen-cell mixture was pipetted into pre-warmed 6-well plates to form one disc per well of approximately $2 \mathrm{~cm}$ in diameter. Plates were then kept at $37^{\circ} \mathrm{C}$ for 30 minutes for collagen I gelation. Next, collagen-containing cell discs were detached from the plate base employing a sterile $1000 \mu$ l plastic tip. $2 \mathrm{ml}$ of complete DMEM medium was added to each well. Macroscopical pictures of the collagen discs were taken every $24 \mathrm{~h}$ to monitor their contraction. Calculation of the disc areas was done by analyzing the corresponding pictures with Fiji (http://rsb.info.nih.gov/ij, $\mathrm{NIH}, \mathrm{USA})$.

\section{Cell migration analysis}

The centroid trajectories of Lgr5-EGFP/RCL-tdT cells were tracked using the Manual Tracking Plug-in in Fiji (http://rsb.info.nih.gov/ij, NIH, USA). Data analysis was performed using a custommade code in Matlab (Mathworks, USA). Cell centroid positions during the experiment were defined as $r_{i}=r(i \Delta t)$, being $\Delta t$ the time between consecutive images and $\mathbf{r}$ a vector. The vector difference between the initial $\left(t=t_{0}\right)$ and the final point $\left(t=t_{f}\right)$ is defined as the displacement vector d and its module $\|\mathbf{d}\|$ as the net displacement. The alignment index of the trajectories is defined as $\cos (2 \cdot \alpha)$, being $\alpha$ the angle between the displacement vector $\mathbf{d}$ and the gap direction, defined as the direction perpendicular to the epithelial edge. This index equals 1 when the trajectory is parallel to the direction of the gap and -1 when it is perpendicular. For the cell migration analysis, $\mathrm{n}_{\text {control }}=136$ cells, $n_{\text {paracrine }}=164$ cells and $n_{\text {physical }}=204$ cells randomly distributed within the epithelia from $\mathrm{N}=3$ independent experiments were analyzed. PIVlab 2.37 (Thielicke and Stamhuis, 2014) was used to quantify the displacements of IECs and ISEMFs on phase contrast time-lapse movies. Briefly, an interrogation window of $23 \mu \mathrm{m}$ with a step of $11.5 \mu \mathrm{m}$ was used to perform Particle Image Velocimetry (PIV) either by FFT window deformation or by ensemble 
correlation. Image sequencing was set as time-resolved and the resulting velocities were filtered using a standard deviation filter $\left(8^{*}\right.$ STD) and a local median filter (threshold $=3$ ). For the PIV analysis, $\mathrm{n}=4$ migration fronts from $\mathrm{N}=2$ independent experiments were used.

\section{Cell orientation analysis}

Orientation fields were obtained using the OrientationJ (Püspöki et al., 2016) plug-in in Fiji. First, images were pre-processed by subtracting the background (40 pixels rolling ball), then a bandpass filter was applied (limits 3 and 40 pixels), and the background noise was subtracted again (40 pixels rolling ball). The resulting images were processed with OrientationJ using a local window of 40 pixels to obtain a structure tensor on a grid of 40 pixels $\times 40$ pixels. With vector fields and their associeated values provided by the plug-in (dominant direction $\omega$, energy $E$ and coherence C) we used a custom-made Matlab code to determine the correlation maps. Briefly, we selected the angles with associated energy $E>0.1$ and coherency $C>0.05$ for each cell type (IEC or ISEMFs) and discarded the rest. These sets of angles were used to calculate the mean cell orientation and the correlation index. To do so, we collected pairs of dominant angles and obtained the difference between them $\theta=$ WIEC - WISEMF for each $x$, y position within the image (note that we only accounted for $\mathrm{x}$, y positions when the dominant angles for both cell types satisfied the conditions for $\mathrm{E}$ and $\mathrm{C}$ ). Then, we defined the correlation index between the two cell types as $\cos (2 \cdot \theta)$, which equals 1 when both cells are oriented parallel to each other and -1 when they are perpendicularly oriented. For this analysis, we used immunostained samples from $\mathrm{N}=2$ independent experiments.

\section{Statistics}

No statistical methods were used to predetermine sample size. Measurements were performed on experimental replicates $(n)$ obtained in different independent experiments (N). Data presentation (as Mean value \pm standard deviation (SD) or as Mean value \pm standard error of the mean (SE)) is defined at the corresponding figure caption. D'Agostino normality test, t-test, and Kruskal-Wallis test were performed using GraphPad Prism 9. Specific values are noted at the corresponding figure captions. 


\section{Acknowledgements}

Funding for this project was provided by a European Union Horizon 2020 ERC grant (agreement no. 647863 - COMIET), the CERCA Programme/Generalitat de Catalunya (2017-SGR-1079), and the Spanish Ministry of Economy and Competitiveness (TEC2017-83716-C2-1-R, and the Severo Ochoa Programme for Centres of Excellence in R\&D 2016-2019). The results presented here only reflect the views of the authors; the European Commission is not responsible for any use that may be made of the information it contains.

Author Contributions: V.F.-M., together with V.A., and A.A.-L. performed the experiments and analysed the data. J.C. designed and performed the image analysis. X.H.-M, and E.B. provided the mice and participated in the discussion. E.M. and V.F.-M. coordinated the project and together with J.C designed the experiments and wrote the paper. All authors have reviewed and approved the content of the manuscript.

\section{References}

Altay, G. et al. (2019) 'Self-organized intestinal epithelial monolayers in crypt and villus-like domains show effective barrier function', Scientific Reports, 9(1), pp. 1-14. doi: 10.1038/s41598019-46497-x.

Altay, G. et al. (2020) 'In vitro Self-organized Mouse Small Intestinal Epithelial Monolayer Protocol', Bio-Protocol, 10(3), pp. 1-21. doi: 10.21769/bioprotoc.3514.

Aoki, R. et al. (2016) 'Foxl1-Expressing Mesenchymal Cells Constitute the Intestinal Stem Cell Niche', Cmgh. Elsevier Inc, 2(2), pp. 175-188. doi: 10.1016/j.jcmgh.2015.12.004.

Attieh, Y. et al. (2017) 'Cancer-associated fibroblasts lead tumor invasion through integrin- $\beta 3$ dependent fibronectin asse', Journal of Cell Biology, 216(11), pp. 3509-3520. doi: 10.1083/jcb.201702033.

Barker, N. et al. (2007) 'Identification of stem cells in small intestine and colon by marker gene Lgr5.', Nature. Nature Publishing Group, 449(7165), pp. 1003-7. doi: 10.1038/nature06196.

Barker, N. (2013) 'Adult intestinal stem cells: critical drivers of epithelial homeostasis and regeneration'. doi: 10.1038/nrm3721.

Barriga, F. M. et al. (2017) 'Mex3a Marks a Slowly Dividing Subpopulation of Lgr5+ Intestinal Stem Cells', Cell Stem Cell, 20(6), pp. 801-816.e7. doi: 10.1016/j.stem.2017.02.007.

Begnaud, S. et al. (2016) 'Mechanics of epithelial tissues during gap closure', Current Opinion in Cell Biology, 42, pp. 52-62. doi: 10.1016/j.ceb.2016.04.006.

Branchfield, K. et al. (2016) 'A three-dimensional study of alveologenesis in mouse lung', Developmental Biology, 409(2), pp. 429-441. doi: 10.1016/j.ydbio.2015.11.017.

Chitturi, R. T. et al. (2015) 'The role of myofibroblasts in wound healing, contraction and its clinical 
implications in cleft palate repair.', Journal of international oral health : JIOH, 7(3), pp. 75-80. Available http://www.ncbi.nlm.nih.gov/pubmed/25878485\%0Ahttp://www.pubmedcentral.nih.gov/articleren der.fcgi?artid=PMC4385733.

Clevers, H. (2013) 'The intestinal crypt, a prototype stem cell compartment.', Cell. Elsevier Inc., 154(2), pp. 274-84. doi: 10.1016/j.cell.2013.07.004.

Desmouliere, A. et al. (1993) 'Transforming growth factor- $\beta 1$ induces $\alpha$-smooth muscle actin expression in granulation tissue myofibroblasts and in quiescent and growing cultured fibroblasts', Journal of Cell Biology, 122(1), pp. 103-111. doi: 10.1083/jcb.122.1.103.

Drost, J. et al. (2015) 'Sequential cancer mutations in cultured human intestinal stem cells', Nature, 521(7550), pp. 43-47. doi: 10.1038/nature14415.

Eyden, B. (2008) 'The myofibroblast: Phenotypic characterization as a prerequisite to understanding its functions in translational medicine: Translational Medicine', Journal of Cellular and Molecular Medicine, 12(1), pp. 22-37. doi: 10.1111/j.1582-4934.2007.00213.x.

Eyden, B., Curry, A. and Wang, G. (2011) 'Stromal cells in the human gut show ultrastructural features of fibroblasts and smooth muscle cells but not myofibroblasts', Journal of Cellular and Molecular Medicine, 15(7), pp. 1483-1491. doi: 10.1111/j.1582-4934.2010.01132.x.

Farin, H. F., Van Es, J. H. and Clevers, H. (2012) 'Redundant sources of Wnt regulate intestinal stem cells and promote formation of paneth cells', Gastroenterology. Elsevier Inc., 143(6), pp. 1518-1529.e7. doi: 10.1053/j.gastro.2012.08.031.

Federici, T. and Boulis, N. M. (1999) 'Myofibroblasts II Intestinal subepithelial myofibroblasts', Invited Review, p. A. doi: 10.1227/01.neu.0000335653.52938.f2.

Fernández-Majada, V. et al. (2016) 'The tumour suppressor CYLD regulates the p53 DNA damage response', Nature Communications, 7. doi: 10.1038/ncomms12508.

Gabbiani, G., Ryan, G. B. and Majno, G. (1971) 'Presence of modified fibroblasts in granulation tissue and their possible role in wound contraction.', New York, pp. 549-550.

Gaggioli, C. et al. (2007) 'Fibroblast-led collective invasion of carcinoma cells with differing roles for RhoGTPases in leading and following cells', Nature Cell Biology, 9(12), pp. 1392-1400. doi: 10.1038/ncb1658.

Gopal, S. et al. (2017) 'Fibronectin-guided migration of carcinoma collectives', Nature Communications, 8. doi: 10.1038/ncomms14105.

Gorono, R. M. R. et al. (1992) 'Radial Keratofomy //. Role of rhe Myofibroblosr in Corneol Wound Conrrocrion', 33(12), pp. 3271-3282.

Gregorieff, A. et al. (2005) 'Expression Pattern of Wnt Signaling Components in the Adult Intestine', Gastroenterology, 129(2), pp. 626-638. doi: 10.1053/j.gastro.2005.06.007.

Harris, A. K., Stopak, D. and Wild, P. (1981) 'Fibroblast traction as a mechanism for collagen morphogenesis', Nature, 290(5803), pp. 249-251. doi: 10.1038/290249a0.

Hinz, B. et al. (2001) 'Alpha-smooth muscle actin expression upregulates fibroblast contractile activity', Molecular Biology of the Cell, 12(9), pp. 2730-2741. doi: 10.1091/mbc.12.9.2730.

Hinz, B. et al. (2007) 'The myofibroblast: One function, multiple origins', American Journal of Pathology, 170(6), pp. 1807-1816. doi: 10.2353/ajpath.2007.070112.

Hinz, B. (2010) 'The myofibroblast: Paradigm for a mechanically active cell', Journal of Biomechanics. Elsevier, 43(1), pp. 146-155. doi: 10.1016/j.jbiomech.2009.09.020.

Hinz, B. and Gabbiani, G. (2003) 'Mechanisms of force generation and transmission by myofibroblasts', Current Opinion in Biotechnology, 14(5), pp. 538-546. doi: 
10.1016/j.copbio.2003.08.006.

Jardé, T. et al. (2018) 'Modelling intestinal carcinogenesis using in vitro organoid cultures', Methods in Molecular Biology, 1725, pp. 41-52. doi: 10.1007/978-1-4939-7568-6_4.

Jester, J. V., Petroll, W. M. and Cavanagh, H. D. (1999) 'Corneal stromal wound healing in refractive surgery: The role of myofibroblasts', Progress in Retinal and Eye Research, 18(3), pp. 311-356. doi: 10.1016/S1350-9462(98)00021-4.

Kabiri, Z. et al. (2014) 'Stroma provides an intestinal stem cell niche in the absence of epithelial Wnts', Development (Cambridge), 141(11), pp. 2206-2215. doi: 10.1242/dev.104976.

Karin, M. and Clevers, H. (2016) 'Reparative inflammation takes charge of tissue regeneration', Nature, 529(7586), pp. 307-315. doi: 10.1038/nature17039.

Khalil, H. et al. (2013) 'Isolation of primary myofibroblasts from mouse and human colon tissue', Journal of Visualized Experiments, (80), pp. 1-6. doi: 10.3791/50611.

Kim, K.-A. et al. (2008) 'R-Spondin family members regulate the Wnt pathway by a common mechanism.', Molecular biology of the cell. American Society for Cell Biology, 19(6), pp. 258896. doi: $10.1091 / \mathrm{mbc}$.E08-02-0187.

Krndija, D. et al. (2019) 'Active cell migration is critical for steady-state epithelial turnover in the gut', 710(August), pp. 705-710.

Lahar, N. et al. (2011) 'Intestinal subepithelial myofibroblasts support in vitro and in vivo growth of human small intestinal epithelium', PLOS ONE, 6(11), pp. 1-9. doi: 10.1371/journal.pone.0026898.

Lee, R. M. et al. (2017) 'Inferring single-cell behaviour from largescale epithelial sheet migration patterns', Journal of the Royal Society Interface, 14(130). doi: 10.1098/rsif.2017.0147.

Lei, N. Y. et al. (2014) 'Intestinal subepithelial myofibroblasts support the growth of intestinal epithelial stem cells', PLOS ONE, 9(1). doi: 10.1371/journal.pone.0084651.

Leushacke, M. and Barker, N. (2014) 'Ex vivo culture of the intestinal epithelium: Strategies and applications', Gut, 63(8), pp. 1345-1354. doi: 10.1136/gutjnl-2014-307204.

McCarthy, N. et al. (2020) 'Distinct Mesenchymal Cell Populations Generate the Essential Intestinal BMP Signaling Gradient', Cell Stem Cell. Elsevier Inc., 26(3), pp. 391-402.e5. doi: 10.1016/j.stem.2020.01.008.

McCarthy, N., Kraiczy, J. and Shivdasani, R. A. (2020) 'Cellular and molecular architecture of the intestinal stem cell niche', Nature Cell Biology. Springer US, 22(9), pp. 1033-1041. doi: 10.1038/s41556-020-0567-z.

McGowan, S. E. et al. (2008) 'Platelet-Derived Growth Factor Receptor-Alpha-Expressing cells localize to the alveolar entry ring and have characteristics of myofibroblasts during pulmonary Alveolar Septal Formation', Anatomical Record, 291(12), pp. 1649-1661. doi: 10.1002/ar.20764.

Mifflin, R. C. et al. (2019) 'Intestinal myofibroblasts : targets for stem cell therapy', (30), pp. 684696. doi: 10.1152/ajpgi.00474.2010.

Parker, A. et al. (2017) 'Cell proliferation within small intestinal crypts is the principal driving force for cell migration on villi', FASEB Journal, 31(2), pp. 636-649. doi: 10.1096/fj.201601002.

Pascal, R. R., Kaye, G. I. and Lane, M.D, N. (1968) 'Colonic Pericryptal Fibroblast Sheath: Replication, Migration, and Cytodifferentiation of a Mesenchymal Cell System in Adult Tissue: I. Autoradiographic studies of normal rabbit colon', Gastroenterology, 54(5), pp. 835-851. doi: 10.1016/S0016-5085(68)80155-6.

Powell, D. W. et al. (2005) 'Epithelial cells and their neighbors: I. Role of intestinal myofibroblasts in development, repair, and cancer', American Journal of Physiology - Gastrointestinal and Liver 
Physiology, 289(1 52-1), pp. 2-7. doi: 10.1152/ajpgi.00075.2005.

Powell, D. W. et al. (2011) 'Mesenchymal Cells of the Intestinal Lamina Propria', Annual Review of Physiology, 73(1), pp. 213-237. doi: 10.1146/annurev.physiol.70.113006.100646.

Püspöki, Z. et al. (2016) 'Transforms and Operators for Directional Bioimage Analysis: A Survey', in Vos, W. H. De (ed.) Focus on Bio-Image Informatics. Springer International Publishing Switzerland, pp. 69-92. doi: 10.1007/978-3-319-28549-8_3.

Reffay, M. et al. (2011) 'Orientation and polarity in collectively migrating cell structures: statics and dynamics.', Biophysical journal. Biophysical Society, 100(11), pp. 2566-75. doi: 10.1016/j.bpj.2011.04.047.

Richman, P. I. et al. (1987) 'Colonic pericrypt sheath cells: Characterisation of cell type with new monoclonal antibody', Journal of Clinical Pathology, 40(6), pp. 593-600. doi: 10.1136/jcp.40.6.593.

Roulis, M. and Flavell, R. A. (2016) 'Fibroblasts and myofibroblasts of the intestinal lamina propria in physiology and disease', Differentiation. Elsevier, 92(3), pp. 116-131. doi: 10.1016/j.diff.2016.05.002.

Sato, T. et al. (2011) 'Long-term expansion of epithelial organoids from human colon, adenoma, adenocarcinoma, and Barrett's epithelium', Gastroenterology, 141(5), pp. 1762-1772. doi: 10.1053/j.gastro.2011.07.050.

Shoshkes-Carmel, M. et al. (2018) 'Subepithelial telocytes are an important source of Wnts that supports intestinal crypts', Nature, 557(7704), pp. 242-246. doi: 10.1038/s41586-018-0084-4.

Terzić, J. et al. (2010) 'Inflammation and Colon Cancer', Gastroenterology, 138(6), pp. 21012114. doi: 10.1053/j.gastro.2010.01.058.

Thielicke, W. and Stamhuis, E. J. (2014) 'PIVlab - Towards User-friendly, Affordable and Accurate Digital Particle Image Velocimetry in MATLAB', Journal of Open Research Software, 2. doi: 10.5334 /jors.bl.

Tomasek, J. J. et al. (2002) 'Myofibroblasts and mechano: Regulation of connective tissue remodelling', Nature Reviews Molecular Cell Biology, 3(5), pp. 349-363. doi: 10.1038/nrm809.

Toshima, M., Ohtani, Y. and Ohtani, O. (2004) 'Three-dimensional architecture of elastin and collagen fiber networks in the human and rat lung', Archives of Histology and Cytology, pp. 3140. doi: 10.1679/aohc.67.31.

Turley, S. J., Cremasco, V. and Astarita, J. L. (2015) 'Immunological hallmarks of stromal cells in the tumour microenvironment', Nature Reviews Immunology. Nature Publishing Group, 15(11), pp. 669-682. doi: 10.1038/nri3902.

Vaughan, M. B., Howard, E. W. and Tomasek, J. J. (2000) 'Transforming growth factor- $\beta 1$ promotes the morphological and functional differentiation of the myofibroblast', Experimental Cell Research, 257(1), pp. 180-189. doi: 10.1006/excr.2000.4869.

Yin, X. et al. (2014) 'Niche-independent high-purity cultures of Lgr5 + intestinal stem cells and their progeny', Nature Methods, 11(1), pp. 106-112. doi: 10.1038/nmeth.2737. 
bioRxiv preprint doi: https://doi.org/10.1101/2021.05.28.446131; this version posted May 29, 2021. The copyright holder for this preprint (which was not certified by peer review) is the author/funder, who has granted bioRxiv a license to display the preprint in perpetuity. It is made available under aCC-BY 4.0 International license.

\section{Figures and Figure Legends}


A

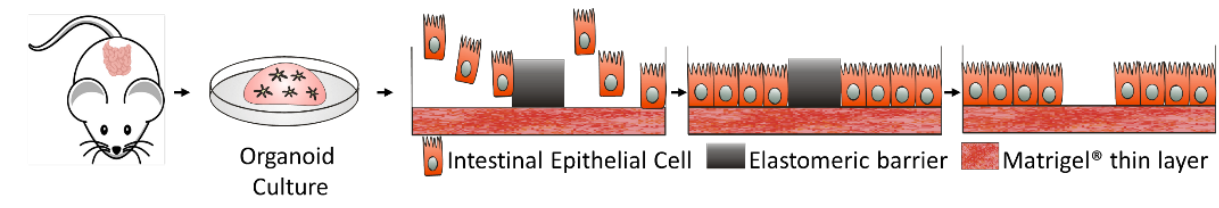

B
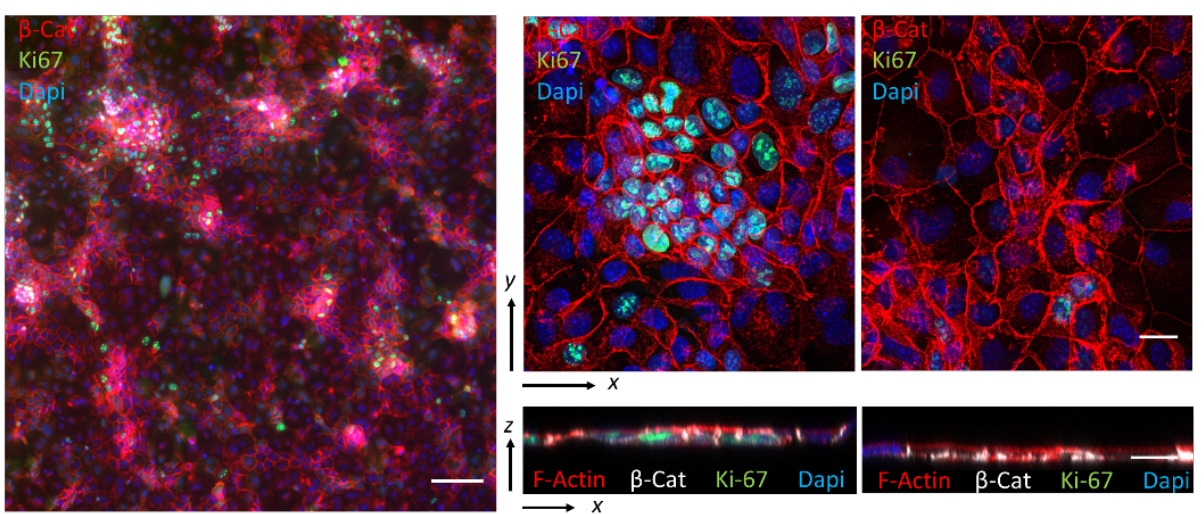

C $\quad \mathrm{t}=\mathrm{Oh}$
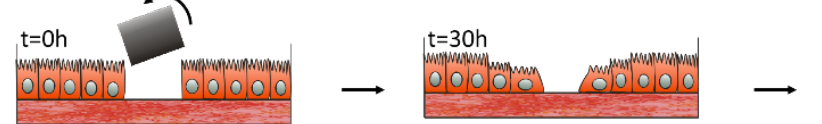

$\mathrm{t}=60 \mathrm{~h}$
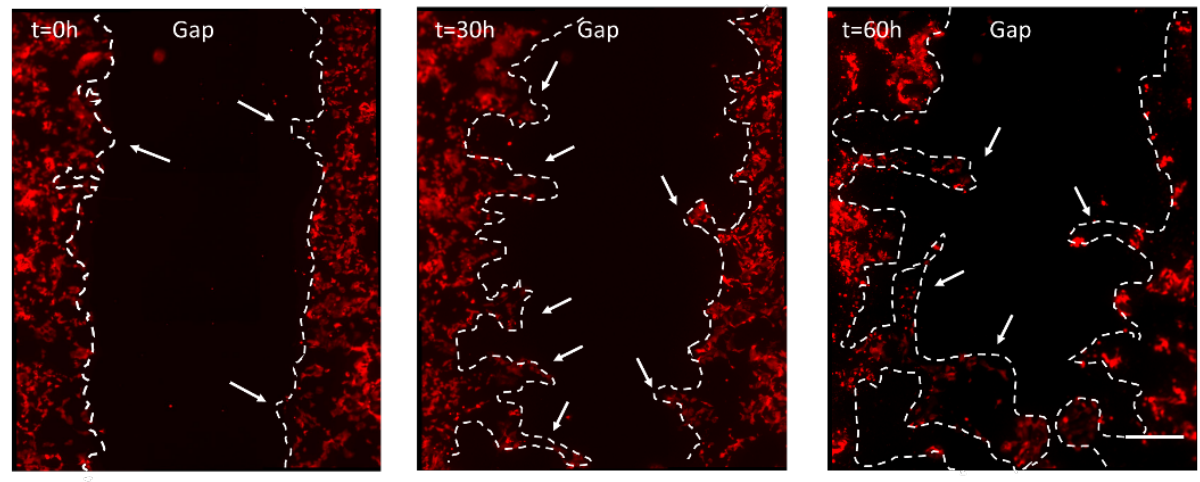

D
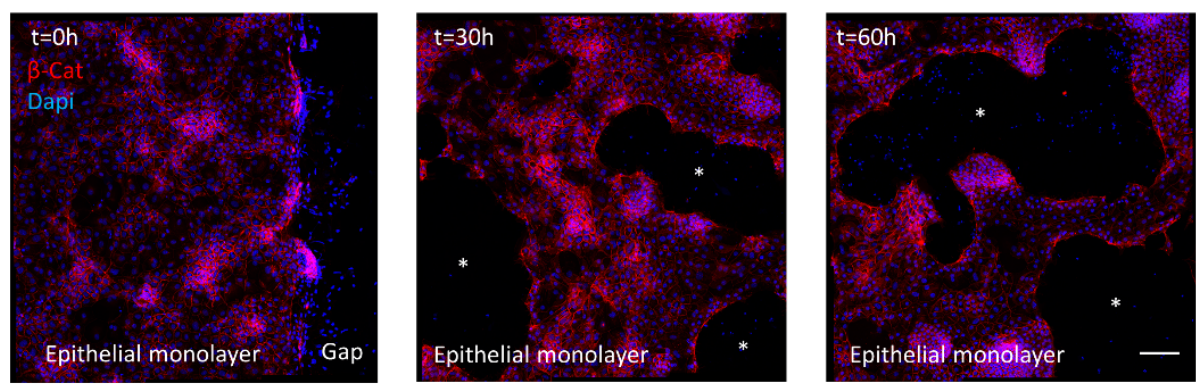

Figure 1. Impaired intestinal epithelial cell migration when only IEC are present in the culture. (A) Scheme depicting the experimental setup. (B) Representative confocal images corresponding to co-immunofluorescence of $\beta$-Catenin ( $\beta$-Cat), Ki67 (left panel and right top panels) and filamentous actin (F-Actin) (right bottom panels) from organoid-derived single cell epithelial monolayers grown on thin films of Matrige ${ }^{\circledR}$ after 3 days in culture. Upper and lower right panels show the top view and the orthogonal cross-sections of the monolayers, respectively. 
Dapi was used to stain the nuclei. Scale bars: $100 \mu \mathrm{m}$ (left panel), $10 \mu \mathrm{m}$ (upper and lower right panels). (C) Live-imaging sequence of tdTomato signal taken 0,30 , and $60 \mathrm{~h}$ after removing the elastomeric barrier from the Lgr5-EGFP/RCL-tdT organoid-derived epithelial cell monolayers. White arrows show the forming epithelial protrusions. Schemes depicting each of the culture conditions are shown. Scale bar: $500 \mu \mathrm{m}$. (D) Representative fluorescence microscope images corresponding to immunofluorescence of $\beta$-Catenin ( $\beta$-Cat) from organoid-derived single cell epithelial monolayers 0,30 , and $60 \mathrm{~h}$ after removing the elastomeric barrier. Dapi was used to stain the nuclei. White asterisks show the forming holes in the epithelial monolayers. Scale bar: $100 \mu \mathrm{m}$.
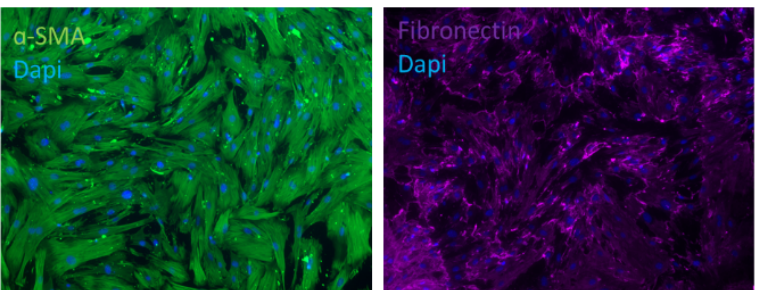

c
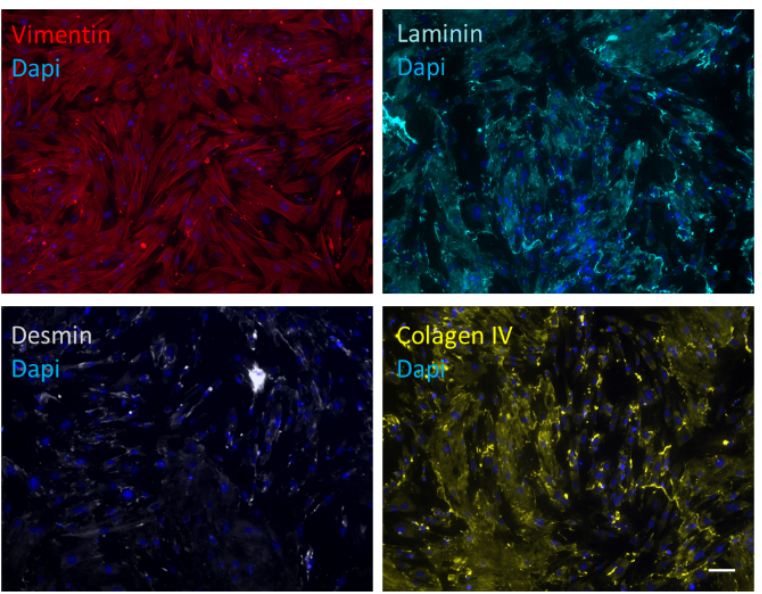

B
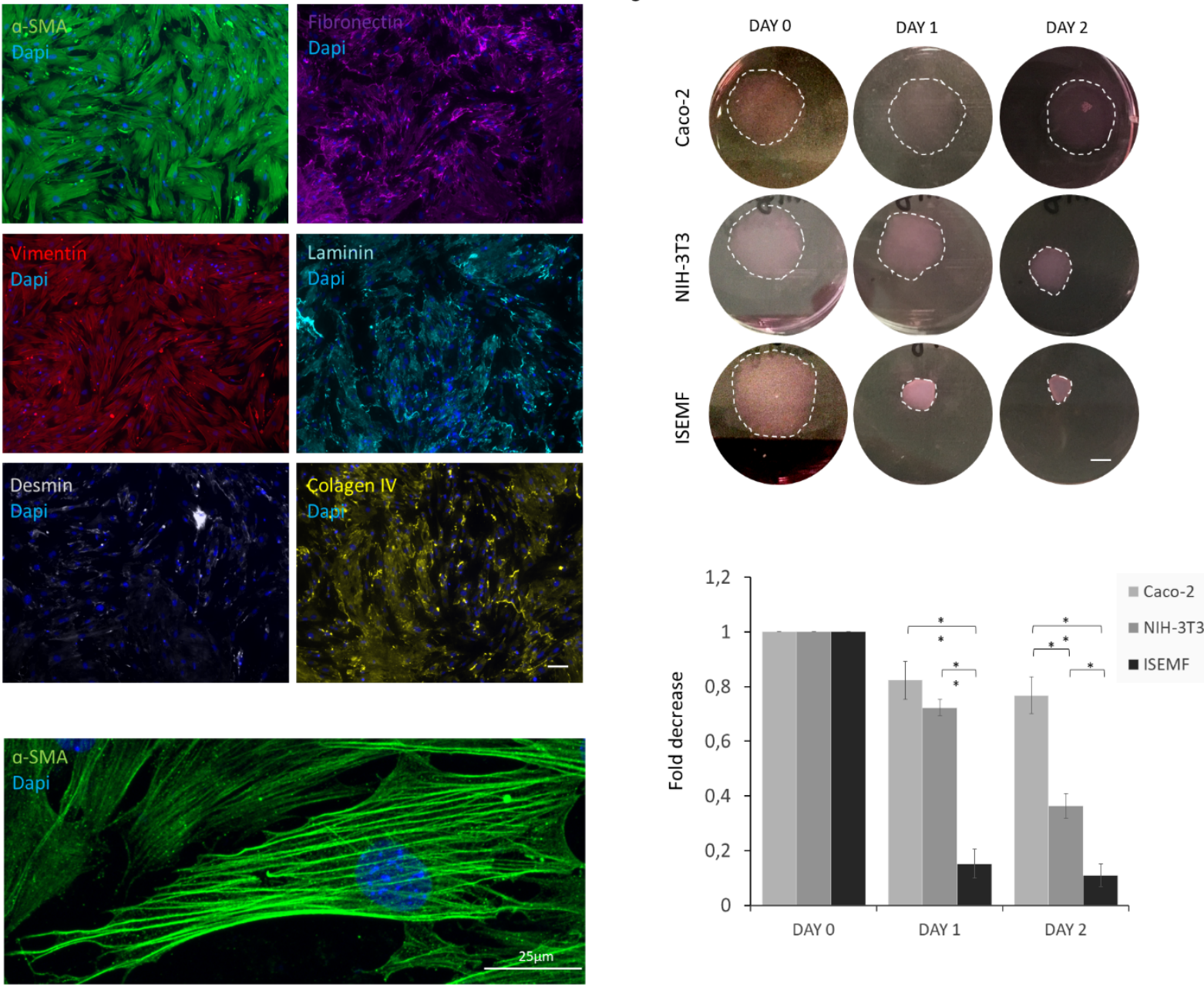

Figure 2. Primary ISEMF characterization. (A) Representative images of immunofluorescence for a-smooth muscle actin (a-SMA), vimentin, desmin, fibronectin, laminin, and collagen IV of 
intestinal subepithelial myofibroblasts (ISEMFs) isolated from mouse gut lamina propria seeded on a thin layer of Matrige ${ }^{\circledR}$. Scale bar: $200 \mu \mathrm{m}$. (B) High magnification immunofluorescence image of a-smooth muscle actin (a-SMA). Scale bar: $25 \mu \mathrm{m}$. In all images Dapi was used to stain the nuclei. (C) Upper panel: pictures of the $3 \mathrm{mg} / \mathrm{ml}$ collagen discs containing Caco-2 cells, NIH-3T3, or ISEMFs 0,1 , and 2 days after embedding them in the collagen discs. For easier identification the collagen discs have been highlighted using a white dotted line. Scale bar: $500 \mu \mathrm{m}$. Lower panel: bar graph showing the mean fold decrease in collagen disc area for each cell type after 0 , 1 , and 2 days $(N=3)$. The data is presented as mean \pm standard deviation (statistics performed through pairwise Student's t-test; significance level set at ${ }^{*} p<0.05$ and ${ }^{* *} p<0.01$ ). 
bioRxiv preprint doi: https://doi.org/10.1101/2021.05.28.446131; this version posted May 29, 2021. The copyright holder for this preprint (which was not certified by peer review) is the author/funder, who has granted bioRxiv a license to display the preprint in perpetuity. It is made available under aCC-BY 4.0 International license.

A

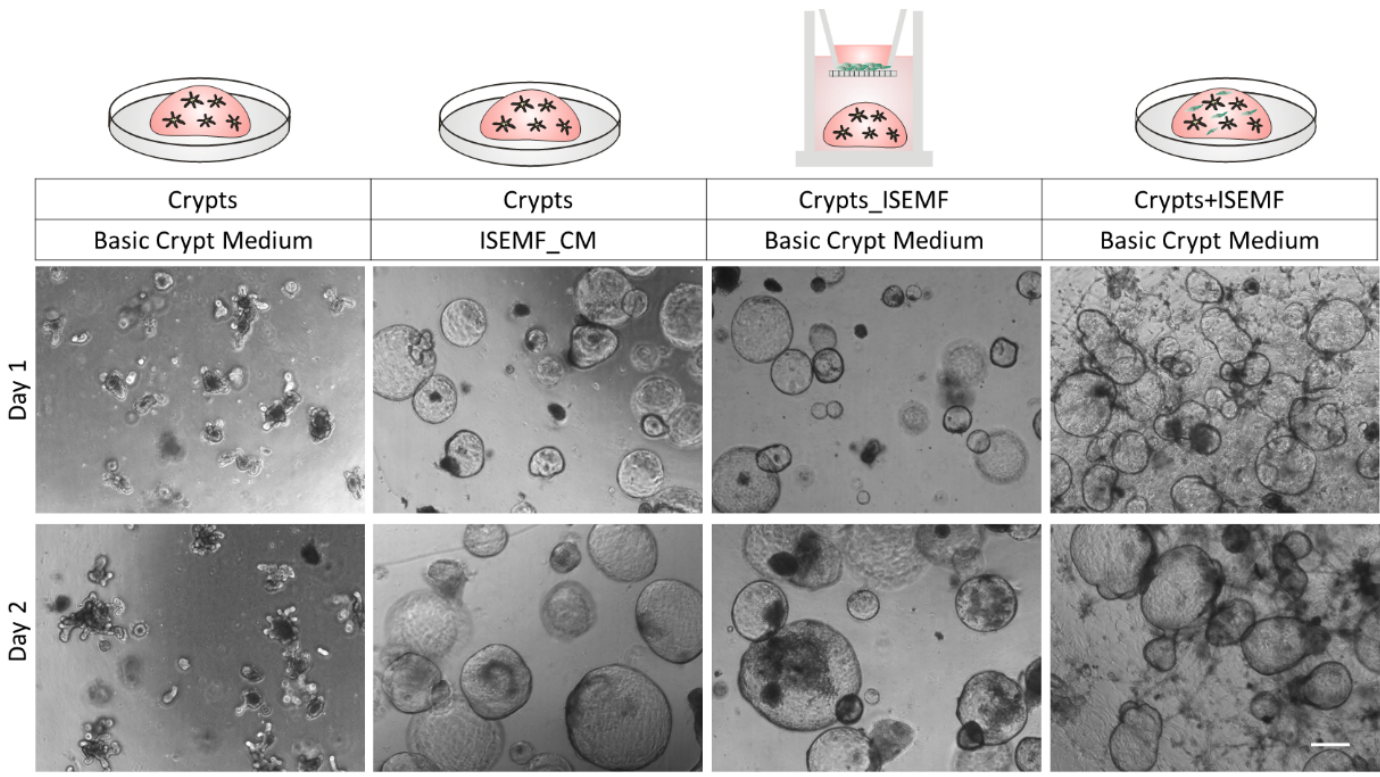

B
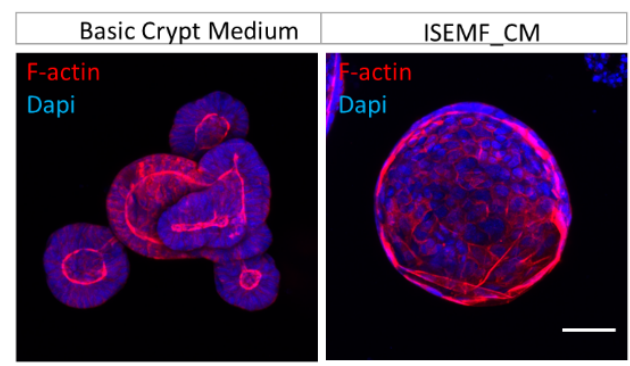

C

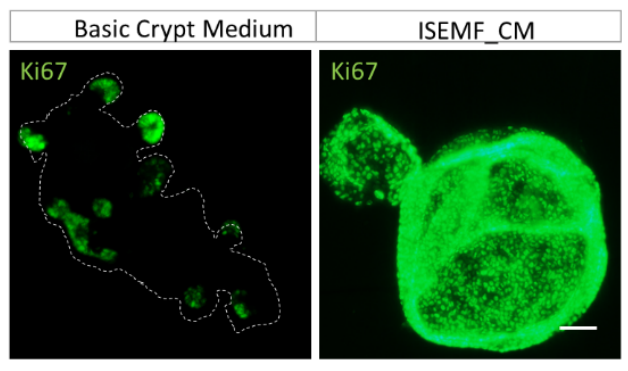

D

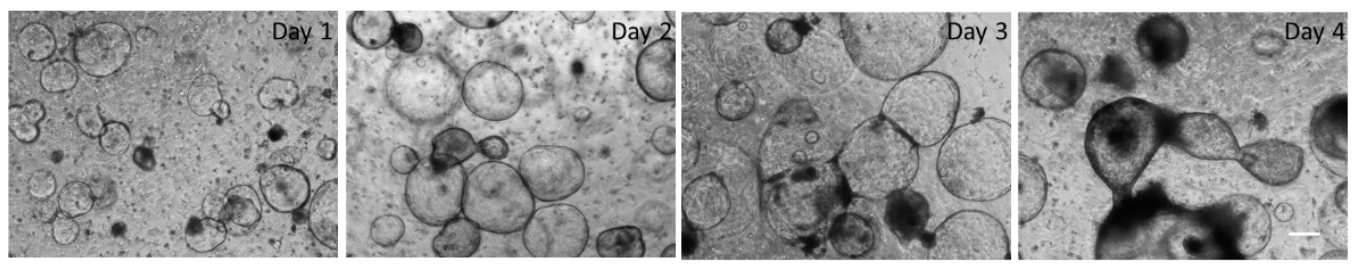

$E$
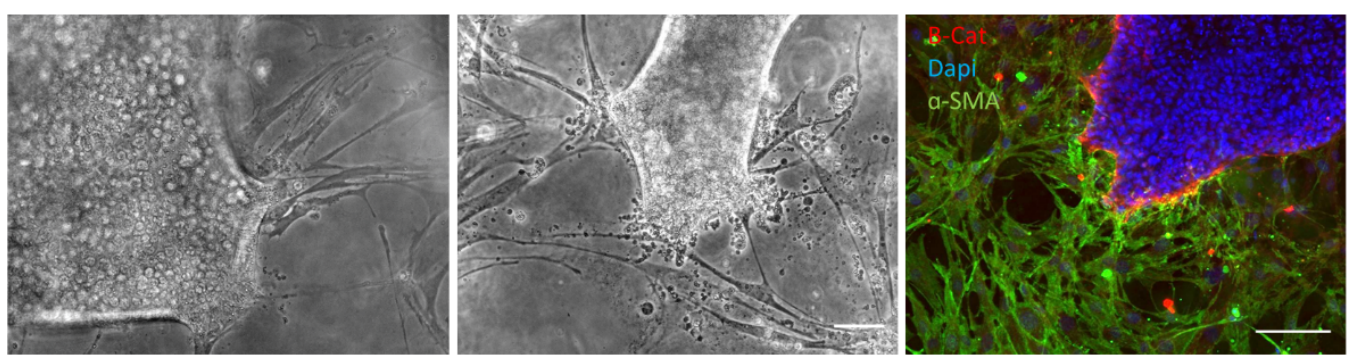

Figure 3. ISEMF-derived paracrine and physical effects on epithelial cell growth. (A) Top

row: Schematics depicting each of the culture conditions. Bottom rows: Representative bright field microscopy images of crypts grown in 3D-Matrige ${ }^{\circledR}$ drops with basic medium, with ISEMFs conditioned medium (ISEMF_CM), in co-culture with ISEMFs seeded on a Transwell ${ }^{\circledR}$ insert, and in a direct physical contact co-culture with ISEMFs after 1 and 2 days in culture. Scale bar: 100 $\mu \mathrm{m}$. Representative confocal images (maximum $Z$ projection) corresponding to 
immunofluorescence of (B) filamentous actin (F-Actin) and (C) Ki67 of intestinal organoids grown with basic medium (left panels) and ISEMF conditioned medium (ISEMF_CM) (right panels). Dapi was used to stain the nuclei. Scale bars: $50 \mu \mathrm{m}$. (D) Representative bright field microscopy images of direct physical contact co-cultures of intestinal organoid cultures and ISEMFs grown in 3D-Matrige $\mathrm{l}^{\circledR}$ drops for 4 consecutive days. Scale bar: $100 \mu \mathrm{m}$. (E) High magnification images corresponding to direct physical contact co-cultures of intestinal organoid cultures and ISEMFs grown in 3D-Matrige ${ }^{\circledR}$ drops for 4 days. Two bright field images (left and midle panels) and an immunofluorescence for $\beta$-catenin ( $\beta$-Cat), alpha-smooth muscle actin (a-SMA) (right panel) are shown. Dapi was used to stain the nuclei. Scale bar: $50 \mu \mathrm{m}$ (bright field images), $100 \mu \mathrm{m}$ (immunofluorescence image). 
bioRxiv preprint doi: https://doi.org/10.1101/2021.05.28.446131; this version posted May 29, 2021. The copyright holder for this preprint

(which was not certified by peer review) is the author/funder, who has granted bioRxiv a license to display the preprint in perpetuity. It is made available under aCC-BY 4.0 International license.

A
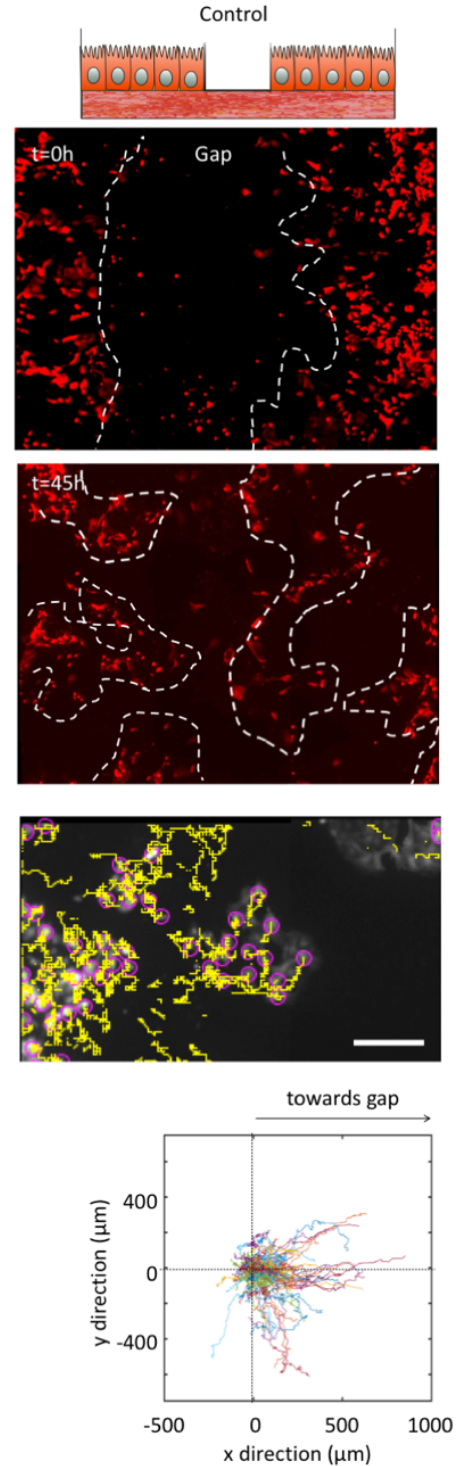

C
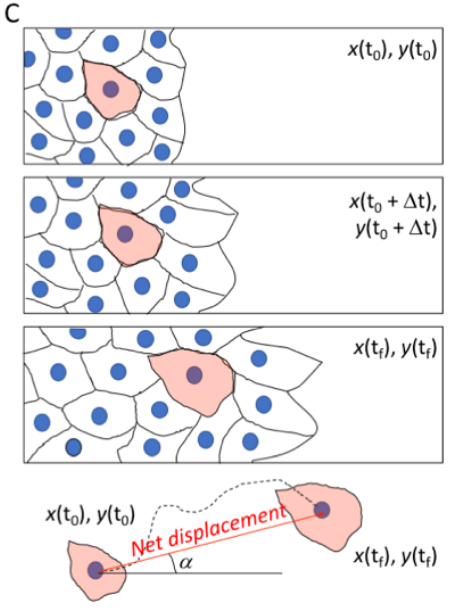

Paracrine
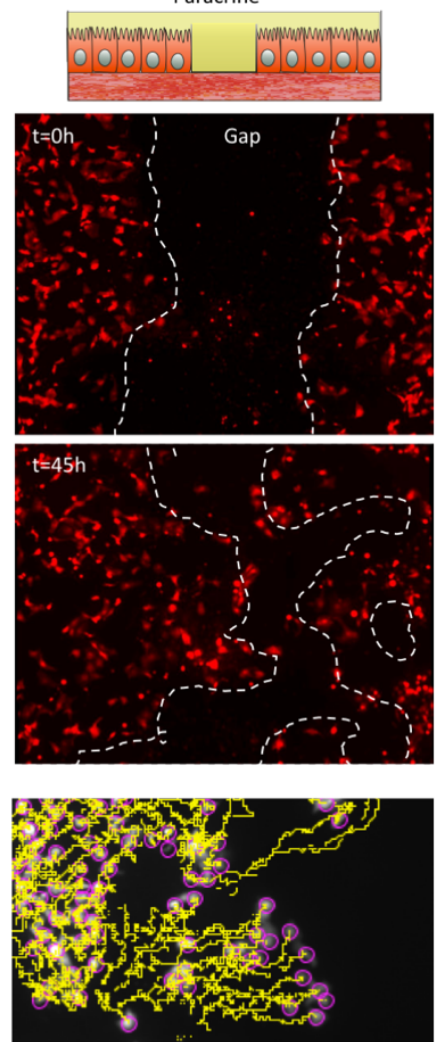

towards gap

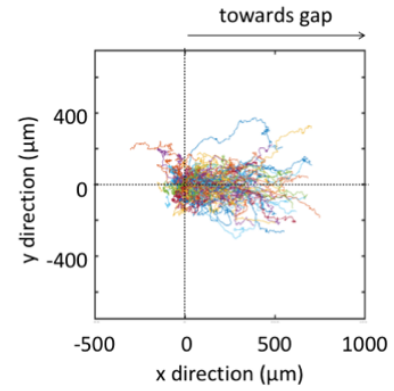

D

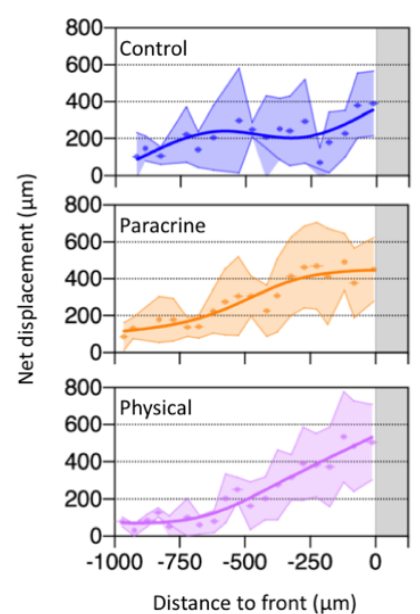

Physical
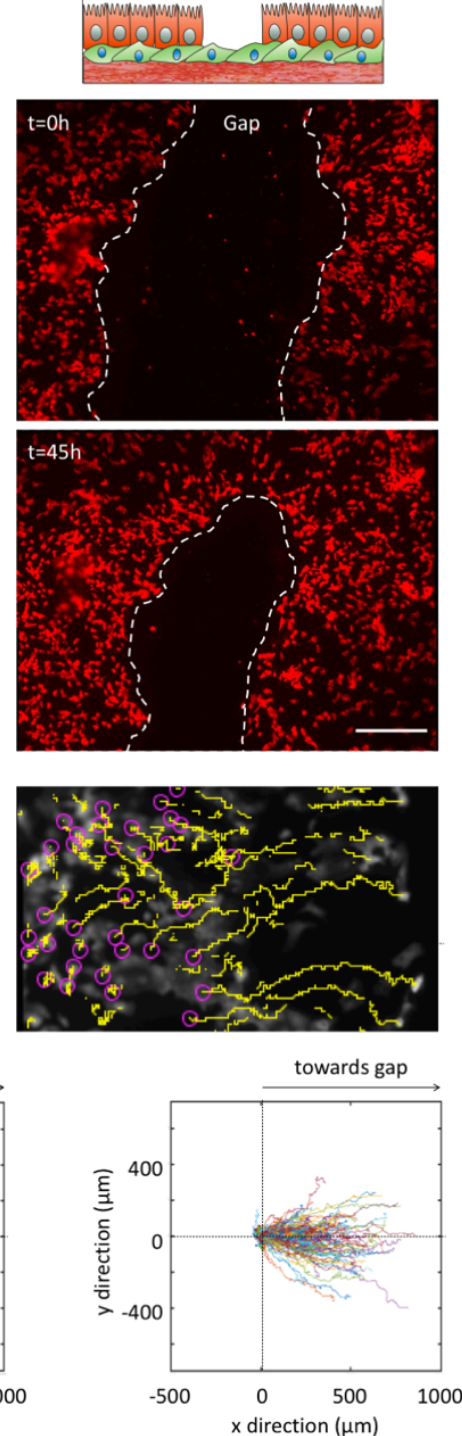

$\mathrm{E}$
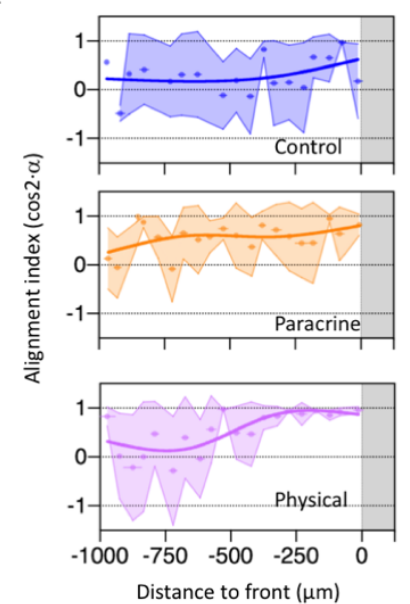

Figure 4. The physical presence of ISEMFs is crucial for an efficient and directed epithelial

migration. (A) Top row: schematics depicting each culture type. Panels below: Live-imaging sequence of tdTomato signal taken 0 and $45 \mathrm{~h}$ after removing the elastomeric barrier from Lgr5- 
EGFP/RCL-tdT organoid-derived epithelial cell monolayer grown on a thin layer of Matrige ${ }^{\circledR}$ and cultured with basic medium (Control), with ISEMFs conditioned medium (Paracrine), or grown on a layer of ISEMFs/Matrige $\left.\right|^{\circledR}$ thin layer with basic medium (Physical). Scale bar: $500 \mu \mathrm{m}$. (B) Top row: individual cell trajectories for each of the culture conditions from (A). Bottom row: trajectories of each individual cell centered at the origin at $t=0$. The direction of the gap is indicated by the arrow. Scale bar: $250 \mu \mathrm{m}$. (C) Schematics of the tracking analysis and the net displacement vector. (D) Net displacement as a function of the initial distance to the migration front for each culture condition. Dots represent the mean value, and the area above and below is defined by the $\mathrm{SD}$, the solid line corresponds to a spline-fitting. Gray region corresponds to the gap outside the epithelia. (E) Alignment index $(\cos (2 \cdot \alpha)$, being $\alpha$ the angle between the net displacement vector and the direction perpendicular to the migration front at $t=0$ ) as a function of the initial distance to the migration front for each culture condition. Dots represent the mean value, and the area above and below is defined by the SD, the solid line corresponds to a spline-fitting. Gray region corresponds to the gap outside the epithelia. $n_{\text {control }}=136$ cells, $n_{\text {paracrine }}=164$ cells and $n_{\text {physical }}=$ 204 cells randomly distributed within the epithelia from $N=3$ independent experiments were analyzed. 
A
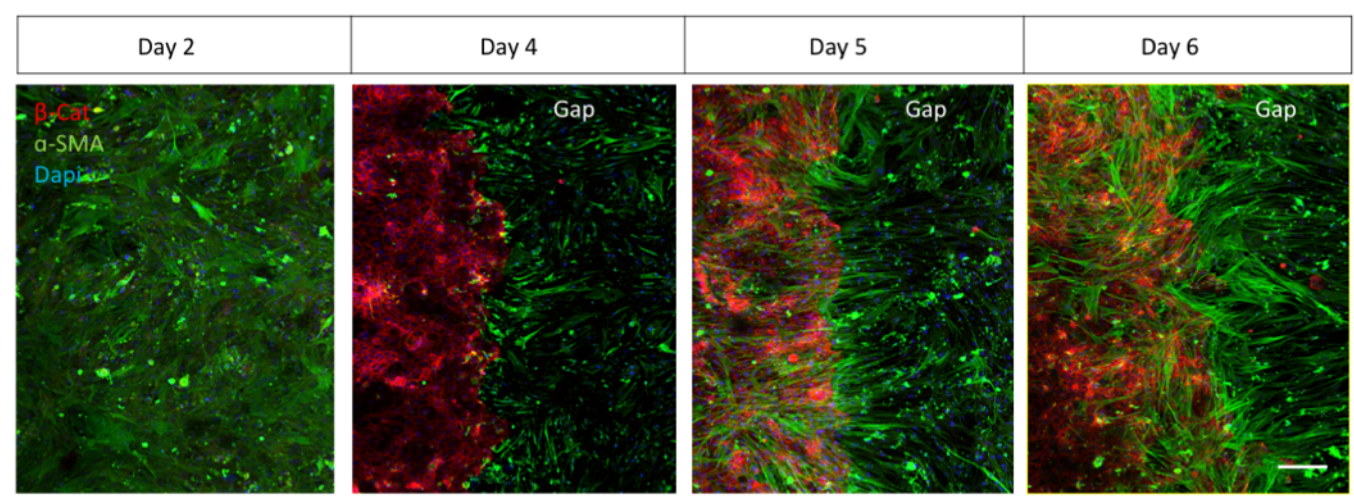

B

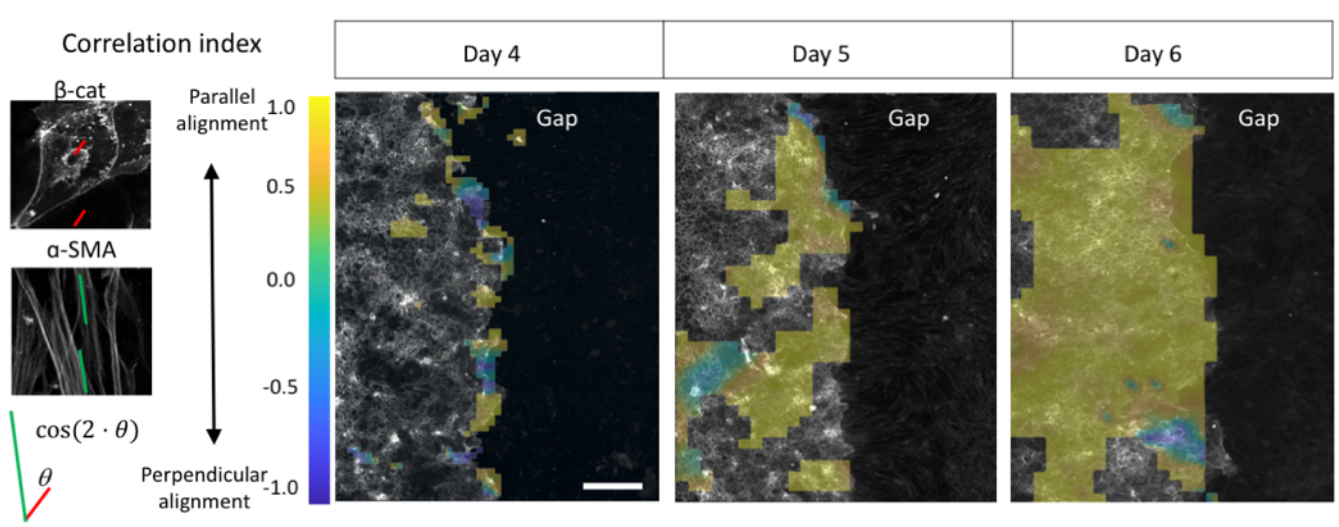

Figure 5. ISEMFs and IECs align at the intestinal epithelial migration front for an active and

collaborative epithelia migration. (A) Representative fluorescent microscopy images corresponding to co-immunofluorescence of $\beta$-Catenin ( $\beta$-Cat) and alpha-smooth muscle actin (a-SMA) of only ISEMFs (left panel) and in co-culture with IECs (middle and right panels) grown on Matrigel ${ }^{\circledR}$ thin layers. Note that the days refer to the number of days in culture. Day 2 shows ISEMFs only while Days 4-6 show the ISEMF/IEC co-culture. The elastomeric barrier was removed on Day 4. Scale bar: $100 \mu \mathrm{m}$. (B) Schematics depicting the dominant directions $(\omega)$ for each cell type and their angular difference $\theta$, from which the correlation index $(\cos (2 \cdot \theta))$ is defined (left panel). Correlation maps at different time points (middle and right panels). Yellow stands for regions with parallel alignment between the two cell types and blue for regions with perpendicular alignment. Regions with no color implies that at least one of the two cell types did not have a defined dominant direction. Scale bar: $500 \mu \mathrm{m}$. Immunostained samples from N = 2 independent experiments were used. 

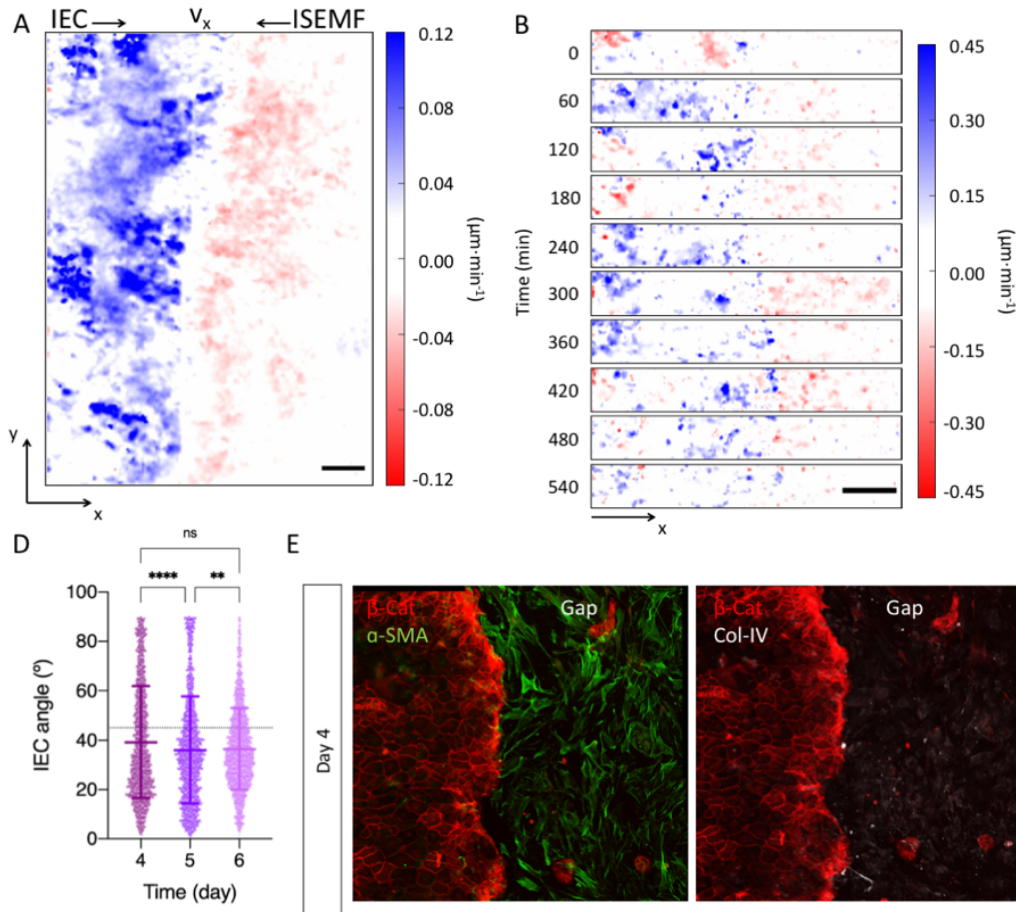

E
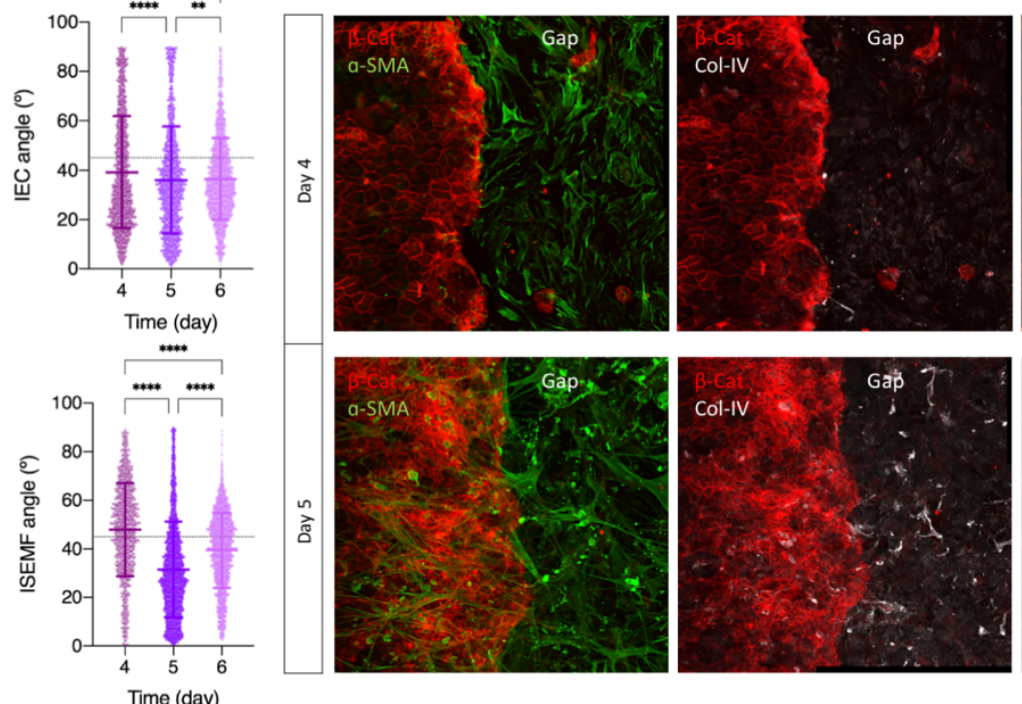

$\mathrm{F}$
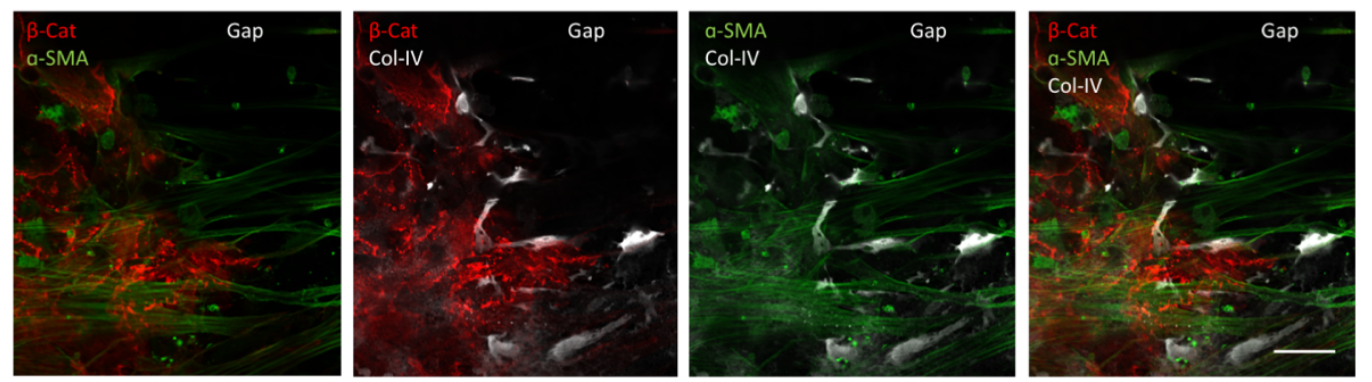

G

a-SMA Col-IV

B-Cat Q-SMA Col-IV

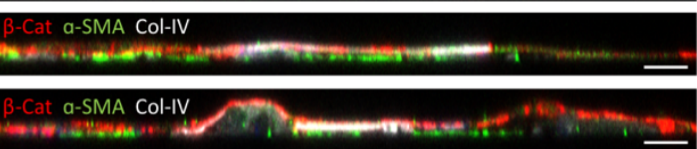

Figure 6. ISEMFs deposit aligned Collagen IV paths to guide IECs movement. (A) Mean velocity $v_{x}$ during the first 9 hours of gap closure. Scale bar: $250 \mu \mathrm{m}$. (B) Snapshots of the instantaneous velocity $v_{x}$ of a region along the first 9 hours of gap closure. Scale bar: $250 \mu \mathrm{m}$. (C) Profiles of the x-component $\left(v_{x}\right)$ and $y$-component $\left(v_{y}\right)$ of the mean velocity across the gap 
direction during the first 9 hours of gap closure. Mean $\pm S D$ of $n=4$ migration fronts of $N=2$ independent experiments. (D) Orientation of IEC and ISEMF cells with respect to the direction perpendicular to the migration front at $t=0$. Mean $\pm S D$ and individual data points are presented. Statistically significant differences were assessed by Kruskal-Wallis test with ${ }^{* *} p<0.01,{ }^{* * *} p<$ 0.001 and $n s p>0.05$. Data was obtained from immunostained samples of $N=2$ independent experiments. (E) Representative fluorescence microscopy images corresponding to coimmunofluorescence of $\beta$-Catenin ( $\beta$-Cat), alpha-smooth muscle actin ( $\alpha-S M A)$, and Collagen-IV (Col-IV) in ISEMFs-IECs co-cultures grown on Matrige ${ }^{\circledR}$ thin layers. The days refer to the number of days in culture and the elastomeric barrier was removed on Day 4. Scale bar: $100 \mu \mathrm{m}$. (F) Higher magnification images from Day 5 of the images shown in (D). Scale bar: $25 \mu \mathrm{m}$. (G) Orthogonal cross-sections on Day 5 of the co-cultures shown in (D). Scale bar: $10 \mu \mathrm{m}$. 
bioRxiv preprint doi: https://doi.org/10.1101/2021.05.28.446131; this version posted May 29,2021 . The copyright holder for this preprint

(which was not certified by peer review) is the author/funder, who has granted bioRxiv a license to display the preprint in perpetuity. It is made available under aCC-BY 4.0 International license.

\section{Supplementary information for}

\section{Subepithelial Myofibroblasts Are Critical Regulators of Intestinal Epithelial}

\section{Restoration}

Vanesa Fernández-Majada*, Jordi Comelles, Verónica Acevedo, Aina Abad-Lázaro,

Xavier Hernando-Momblona, Eduard Batlle, Elena Martinez*

E-mail address: emartinez@ibecbarcelona.eu; vfernandez@ibecbarcelona.eu 
bioRxiv preprint doi: https://doi.org/10.1101/2021.05.28.446131; this version posted May 29, 2021. The copyright holder for this preprint

(which was not certified by peer review) is the author/funder, who has granted bioRxiv a license to display the preprint in perpetuity. It is made available under aCC-BY 4.0 International license.

\section{Supplementary figures}

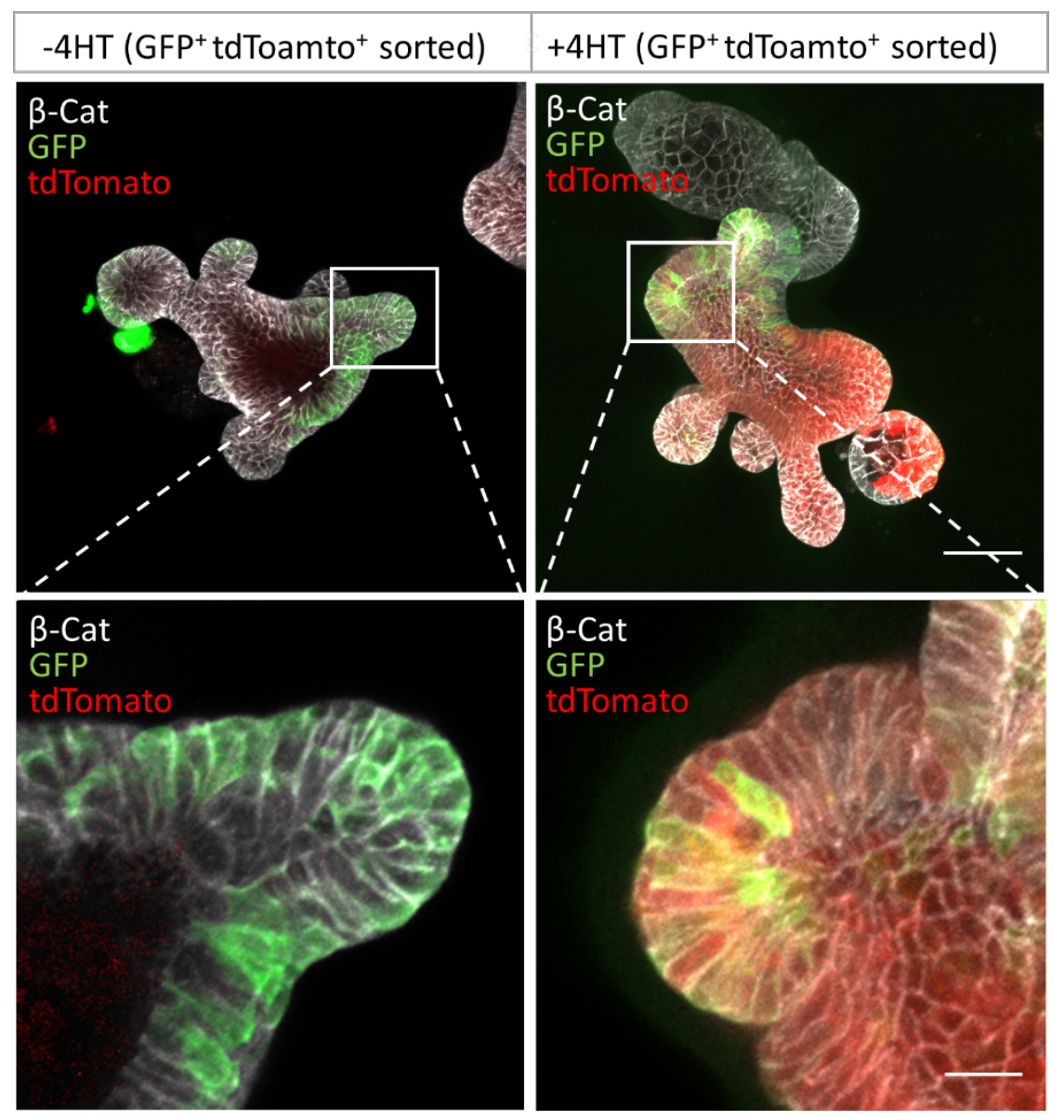

Figure 1 - Figure Supplement 1. Lgr5-EGFP/RCL-tdT organoid characterization. (A)

Representative confocal images ( $Z$ projections) showing GFP and tdTomato signal and $\beta$-Catenin ( $\beta$-Cat immunofluorescence of Lgr5-EGFP-IRES-creERT2/RCL-tdT-derived organoids either after 48h treatment with 4-Hydroxitamoxifen (4-HT) (right panels) or untreated (left panels). The treated organoids were digested to single cells sorted for $\mathrm{GFP}^{+}$and tdTomato ${ }^{+}$and seeded in Matrige ${ }^{\circledR}$ drops to obtain the organoids shown in the figure: Lgr5-EGFP-IRES-creERT2/RCL-tdT (left panels) and Lgr5-EGFP/RCL-tdT (right panels). The lower panels show magnifications of the organoid crypt regions indicated in the upper panels. Scale bars: $50 \mu \mathrm{m}$ (upper panels) and 12 $\mu \mathrm{m}$ (lower panels). 

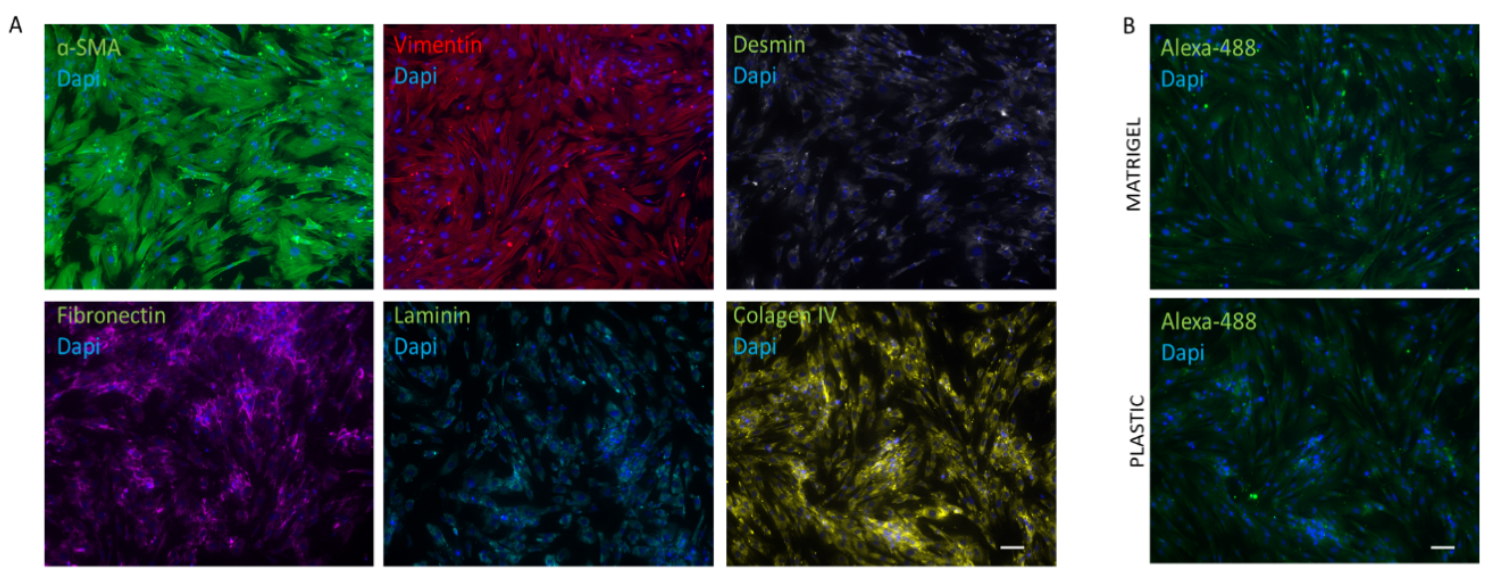

Figure 2 - Figure Supplement 1. Primary ISEMFs characterization. (A) Representative images showing the immunofluorescence for a-smooth muscle actin (a-SMA), vimentin, desmin, fibronectin, and collagen IV of intestinal subepithelial myofibroblasts (ISEMFs) isolated from mouse gut lamina propria seeded on regular non-coated plastic tissue plates. (B) Representative images showing secondary antibody control (Alexa-488) immunofluorescence of ISEMFs seeded either on a thin film of Matrigel ${ }^{\circledR}$ (upper panel) or on a non-coated plastic tissue plate (lower panel). In all images Dapi was used to stain the nuclei. Scale bars: $100 \mu \mathrm{m}$. 
bioRxiv preprint doi: https://doi.org/10.1101/2021.05.28.446131; this version posted May 29, 2021. The copyright holder for this preprint

(which was not certified by peer review) is the author/funder, who has granted bioRxiv a license to display the preprint in perpetuity. It is made available under aCC-BY 4.0 International license.

A
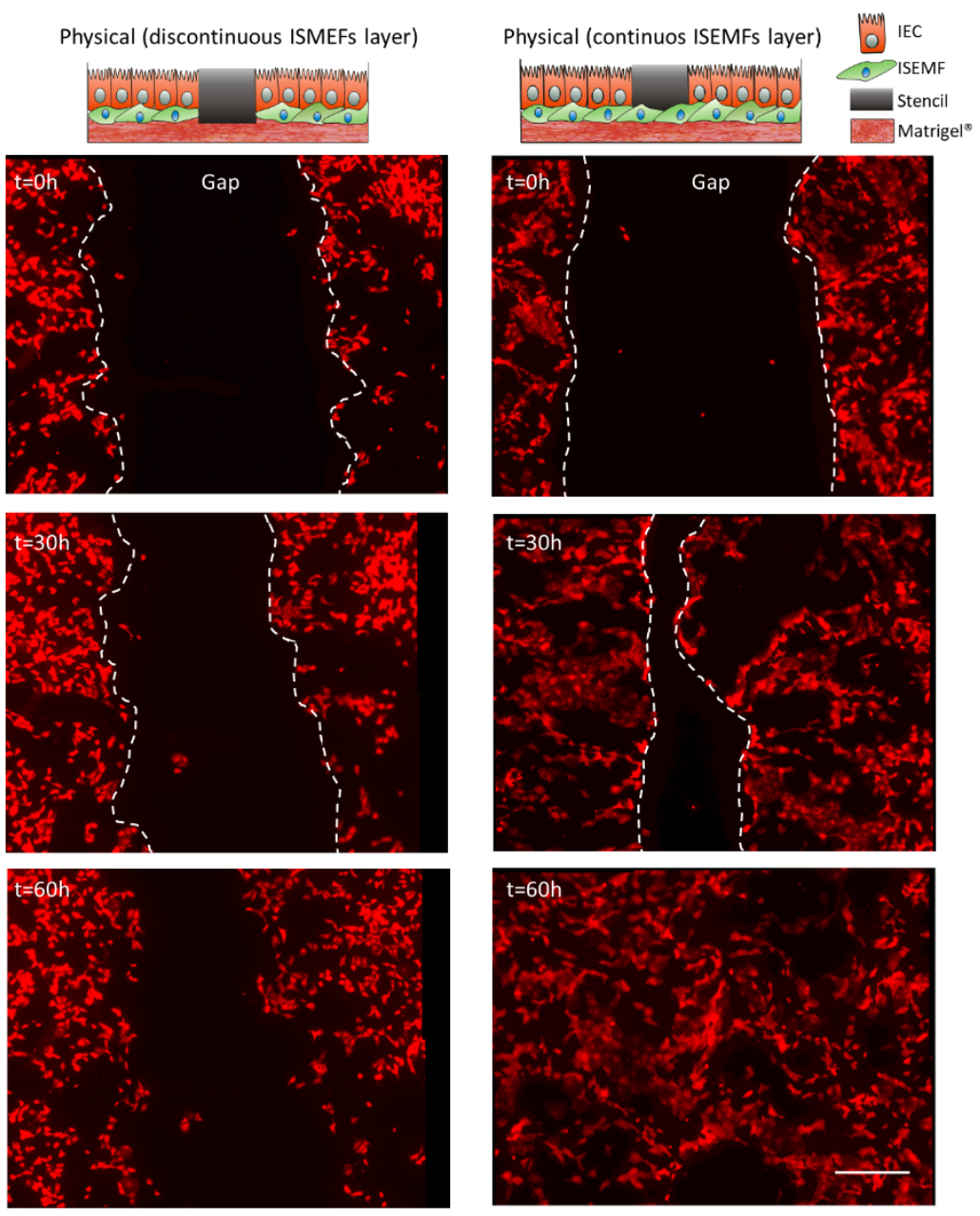

B
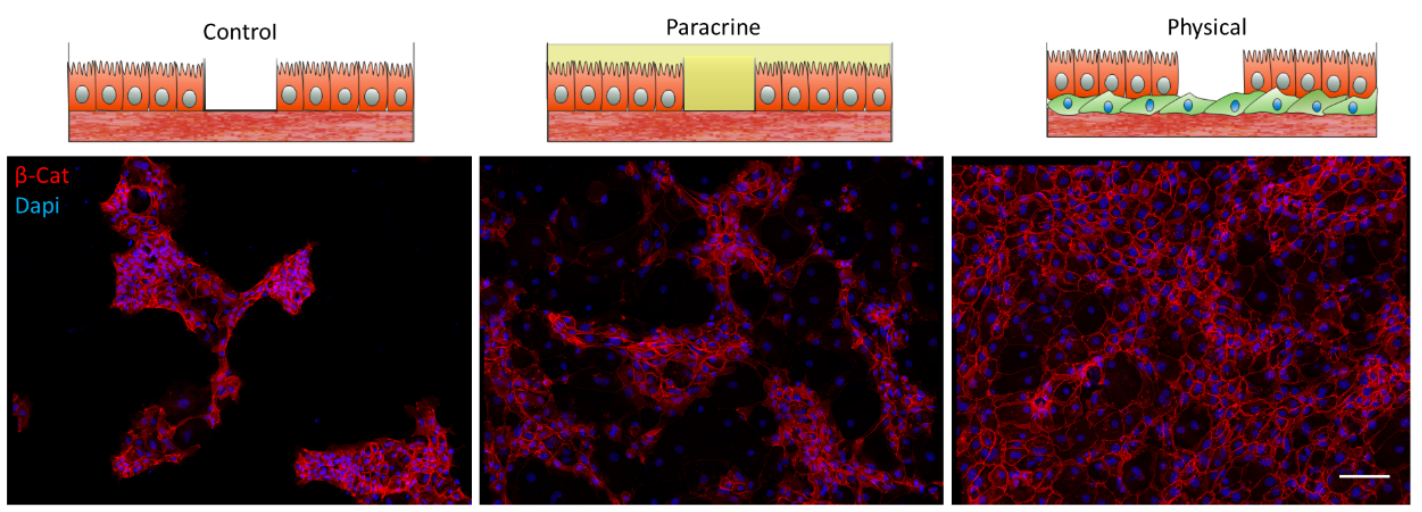

Figure 4 - Figure Supplement 1. Physical interaction between IEC and a continuous layer of ISEMFs is required for an efficient IEC migration and a cohesive maintenance of the epithelial tissue (A) Top row: Schematics depicting each of the culture conditions. Panels: Liveimaging sequence of tdTomato signal corresponding to 0,30 and 60 hours $(\mathrm{h})$ after removing the elastomeric barrier from Lgr5-EGFP/RCL-tdT organoid-derived epithelial cells monolayer grown on a discontinuous (left panels) or continuous (right panels) layer of ISEMFs layer and cultured with basic medium. (B) Top row: Schematics depicting each of the culture conditions. Panels: 
bioRxiv preprint doi: https://doi.org/10.1101/2021.05.28.446131; this version posted May 29, 2021. The copyright holder for this preprint

(which was not certified by peer review) is the author/funder, who has granted bioRxiv a license to display the preprint in perpetuity. It is made available under aCC-BY 4.0 International license.

Representative fluorescence microscope images corresponding to immunofluorescence of $\beta$ Catenin ( $\beta$-Cat) 45 hours $(\mathrm{h})$ after removing the elastomeric barrier from organoid-derived epithelial cells monolayers grown on a thin layer of Matrige ${ }^{\circledR}$ and cultured either with basic medium (left panels, Control) or with ISEMF conditioned medium (ISEMF-CM) (middle panels, Paracrine), or grown on a layer of ISEMFs / Matrigel ${ }^{\circledR}$ thin layer with basic medium (right panels, Physical). Dapi was used to stain the nuclei. Scale bar: $100 \mu \mathrm{m}$. 
A

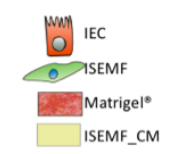

B

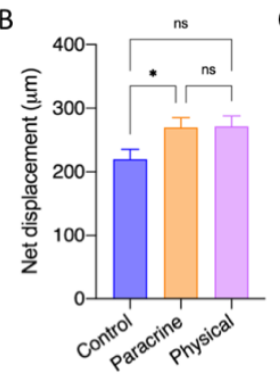

D

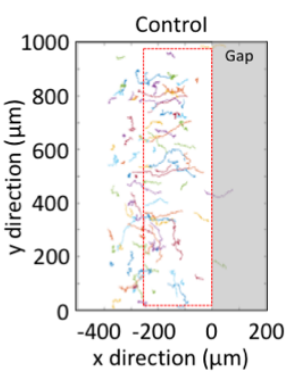

Control

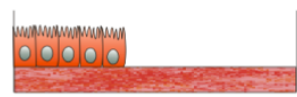

C

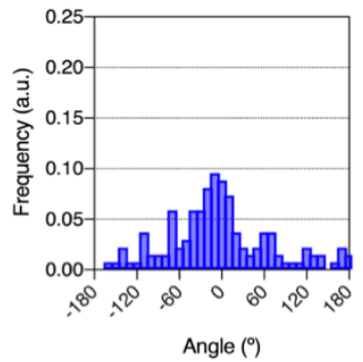

Paracrine
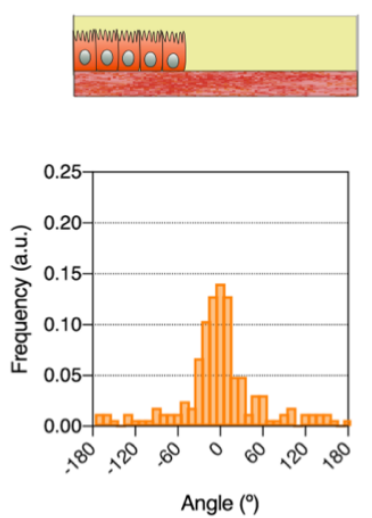

E
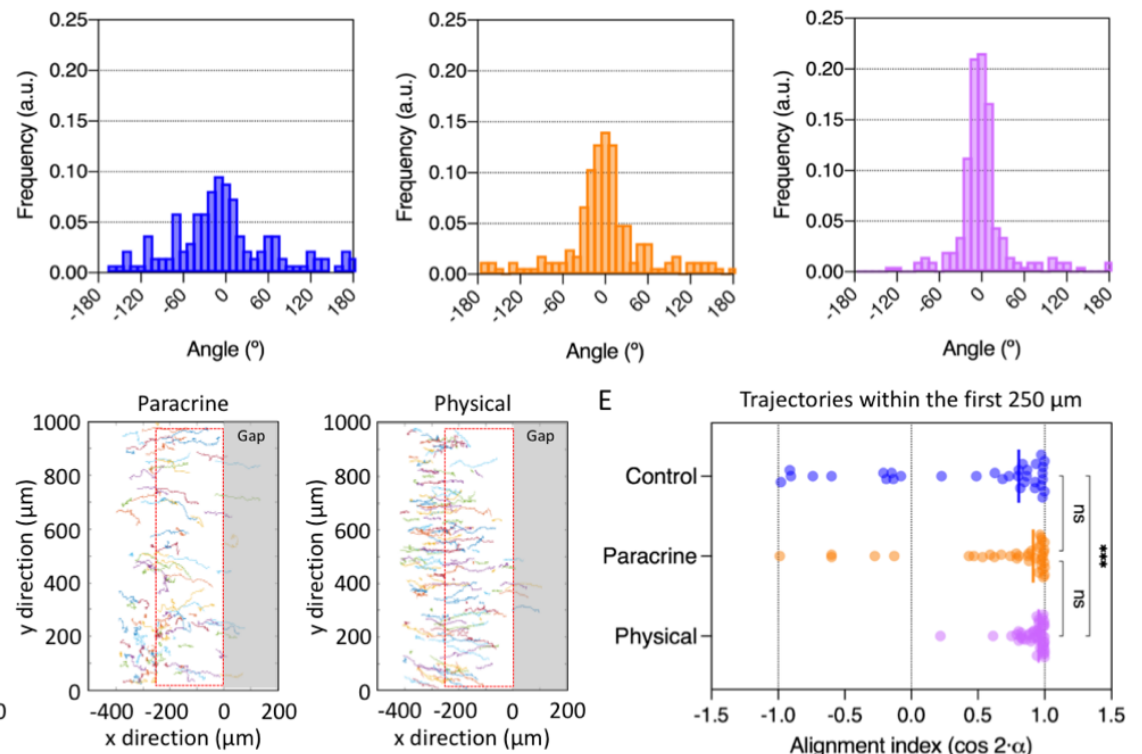

Figure 4 - Figure Supplement 2. Physical presence of ISEMFs increases the net displacement and the orientation toward the gap of IECs trajectories. (A) Scheme depicting the different culture conditions. (B) Net displacement for each of the culture conditions. Mean \pm SEM of $n_{\text {control }}=136$ cells, $n_{\text {paracrine }}=164$ cells and $n_{\text {physical }}=204$ from $\mathrm{N}=3$ independent experiments. Statistically significant differences were assessed by Kruskal-Wallis test with * $p<$ 0.05 and ns $p>0.05$. (C) Distribution of the angle between the displacement vector and the direction perpendicular to the migration front for each of the culture conditions. (D) Cell trajectories for the different experimental conditions. The grey region corresponds to the gap while the red rectangle highlights the first $250 \mu \mathrm{m}$ of the epithelia behind the migration front. (E) Alignment index for cells' trajectories within the first $250 \mu \mathrm{m}$ behind the direction front. Mean and individual values of $n_{\text {control }}=33$ cells, $n_{\text {paracrine }}=38$ cells and $n_{\text {physical }}=46$ from $\mathrm{N}=3$ independent experiments. Statistically significant differences were assessed by Kruskal-Wallis test with ${ }^{* * *} p<0.001$ and ns $p>0.05$. 
A

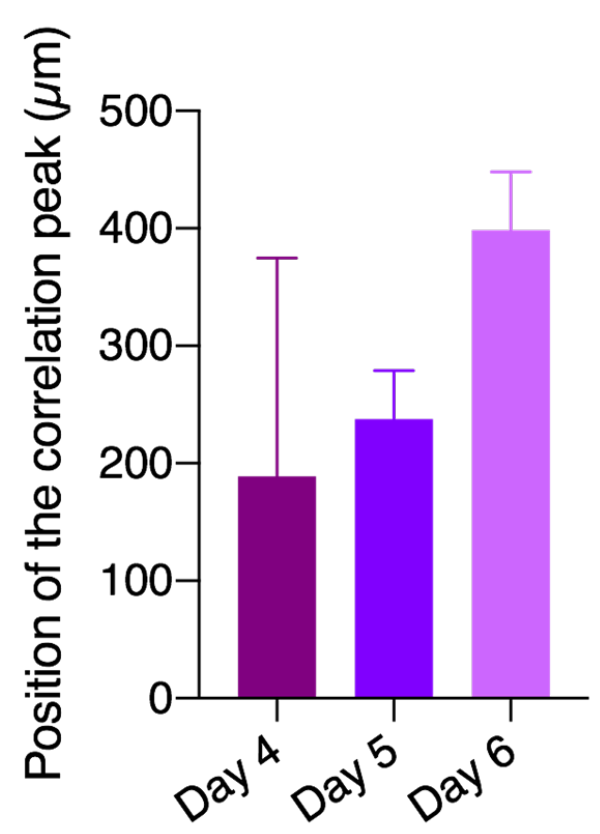

B

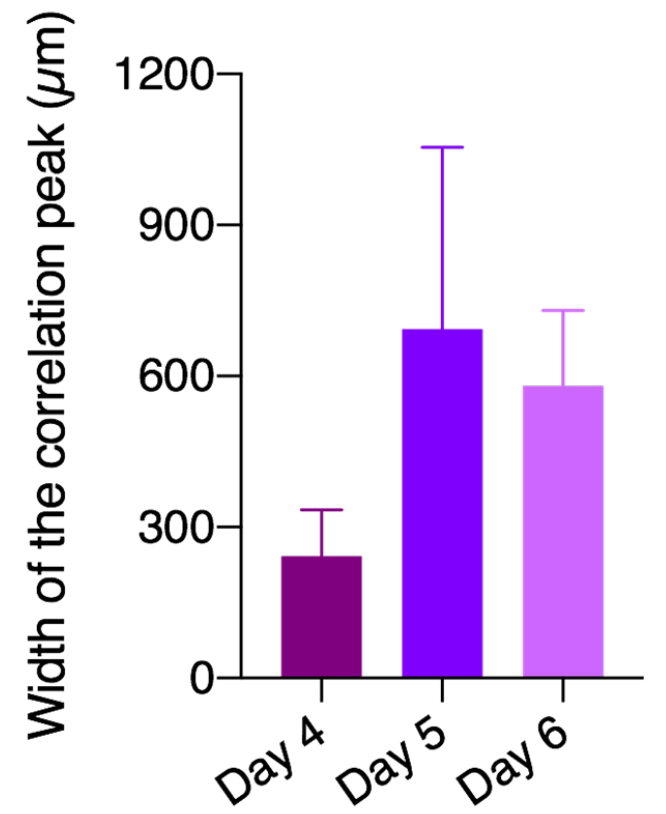

Figure 5 - Figure Supplement 1. Correlation peak's position and width increase with time.

Mean \pm SD of $(A)$ the position of the correlation peak for different time points and $(B)$ the Mean \pm $\mathrm{SD}$ of the width of this correlation peak. Data from immunostained images of $\mathrm{N}=2$ experiments. 

made available under aCC-BY 4.0 International license.

Video 1. Gap closure experiment in 'control' condition. tdTomato signal from the Lgr5-EGFP/RCL$t d T$ organoid-derived epithelial cell monolayers. Time hh:mm. Scale bar: $250 \mu \mathrm{m}$.

Video 2. Intestinal crypts grown in direct contact with ISEFMs perform contraction-relaxation cylces. Time hh:mm.

Video 3. ISEMFs physically interact with the intestinal cysts. Time hh:mm.

Video 4. Gap closure experiment in 'paracrine' condition. tdTomato signal from the Lgr5EGFP/RCL-tdT organoid-derived epithelial cell monolayers. Time hh:mm. Scale bar: $250 \mu \mathrm{m}$.

Video 5. Gap closure experiment in 'physical condition'. tdTomato signal from the Lgr5EGFP/RCL-tdT organoid-derived epithelial cell monolayers. Time hh:mm. Scale bar: $250 \mu \mathrm{m}$. 